\title{
Über die Sätze vom Parallelogramm der Kräfte und vom Hebel; so wie vom Parallelepipedum der Kräfte.
}

\author{
(Vom Herausgeber.)
}

V o r b e m e r k u n g.

1.

Der Satz vom Parallelogramm der Kräfle läfst sich auf den Satz com Hebel gründen, also mitlelbar beweisen; wie es z. B. Poinsot in seiner Statik versuchte. Eben so läfst sich umgekehrt der Satz vom Hebel auf den vom Parallelogramm der Kräfte gründen, also ebenfalls mittelbar beweisen; wie es z. B. Eylelwein in seiner Statik gethan hat. Auch fehlt es nicht an mancherlei unmittelbaren Beweisen des einen und des andern Satzes, unabhängig von einander. Die unmittelbaren Beweise des Satzes vom Hebel sind einfach, und bedürfen nur gewöhnlich des Überganges vom Rationalen zum Irrationalen. Diejenigen vom Parallelogramm der Kräfle sind zum Theil sehr künstlich, und beschreiten gewöhnlich ebenfalls jenen Übergang. Kürzlich hat Möbius den unabhängigen Beweisen des Satzes vom Parallelogramm der Kräfte noch einen neuen, sehr sinnreichen Beweis hinzugefügt, welchen auch dieses Journal im 2ten Heft 42ten Bandes (S. 179 etc.) mittheilte.

Wir wollen hier versuchen, die mittelbaren Beweise der beiden Sätze, die man für die Elemente gewöhnlich vorzieht, noch etwas schärfer und einfacher zu geben; dann aber andere, unmitlelbare Beweise der beiden Sätze anheimstellen, aus deren jedem der andere folgt. Endlich werden wir einige Bemerkungen über den Satz vom Parallelepipedum der Krüfte beifügen.

Da ein so ausgezeichneter Mathematiker wie Möbius noch neuerdings es nicht verschmäht hat, sein Nachdenken auf diese elementaren Dinge zu richten, so wird es dem Herausgeber dieses Journals erlaubt sein, auch seine Ansichten darüber hier möglichst lurz mitzutheilen. In der That ist die möglichșt feste und einfache Begründung der Mathematik nicht minder wichtig, als die immer weitere Entwicklung derselben. Auch ist sie wohl eben so schwierig, als 'diese, ja selbst eben so uninöglich, als der Abschlufs der 
weitern Entwicklung. Die Mathematik bewegt sich, gleich allem menschlichen Wissen, zwischen zwei unbegrenzten Unendlichen. Sie hebt sich aus der unergründlichen Tiefe des einen Unendlichen hervor, und strebt, immer weiter sich entwickelnd, nach oben zu einem andern Unendlichen. Beide sind unbegrenzt. In der Geometrie z. B. sucht man vergebens seit Jahrtausenden nach einer genügenden Theorie der Parallelen; und eine Erklärung der Ebene, die strenge und zugleich für das Weitere brauchhar wäre, giebt es nicht, obgleich schon die Elemente sich überall in der Ebene bewegen. Sie sprechen immerfort von der Ebene, und sagen doch nicht deutlich, was sie eigentlich sei. Wenn Jemand sagt: die Geometrie habe keinen sichern Grund, sondern müsse wohl nur deshalb richtig sein, weil alle ihre, auf den vorausgesetzten Grund aufgeschichteten strengen Schlüsse nirgends auf Widersprüche führen, so dürfte sich dagegen nicht viel einwenden lassen. Daher möge man denn auch fortgesetzte Bemühungen um die möglichst feste, wenigstens klare und einfache Begründung der Elemente nicht verwerfen und nicht etwa gleichgültig darauf hinabsehen.

Beweis des Satzes vom Parallelogramm der Kräfte, wenn der Satz vom Hebel als bewiesen vorausgesetzt wird.

2.

A. Der Satz vom Hebel besagt, dafs zwei Kräfte $\boldsymbol{p}$ und $q$, die in beliebigen, aber parallelen Richtungen $\boldsymbol{A A}_{2}$ und $\boldsymbol{B B}$ (Fig. 1, 'Taf. III.) auf den Hebel $\boldsymbol{A C B}$ wirken, um denjenigen Punct $\boldsymbol{C}$ im Gleichgewicht sind, dessen Entfernungen $\boldsymbol{C A}=a$ von $\boldsymbol{A}$ und $\boldsymbol{C B}=\boldsymbol{b}$ von $\boldsymbol{B}$ sich verhalten, wie $q$ zu $\boldsymbol{p}$, so dafs

$$
\text { (1.) } \quad a p=b q \text {. ist. }
$$

B. Wenn man von Kräften $p$ und $q$ spricht, so meint man unter den Buchstaben $\boldsymbol{p}$ und $q$ ganze oder gebrochene, rationale oder irrationale Zahlen, deren Einheit irgend eine zur Einheit angenommene, Kraft ist. Eben so, wenn man von den Entfernungen $a$ und $b$ des Puncts $\boldsymbol{C}$ von $\boldsymbol{A}$ und $\boldsymbol{B}$ spricht, so meint man unter den Buchstaben $a$ und $b$ ganze oder gebrochene, rationale oder irrationale Zahlen, deren Einleit irgend eine zur Einheit angenommene Länge der geraden Linie ist.

Offenbar ist es aber ganz gleichgültig, welche Kraft man zur Einheit der. Kräfte und welche Linienlänge man zur Einheit der Linienlänge setzt, wenn man nur stets dieselben Einheiten beibehält. 
C. Hat man nun für die auf den Hebel wirkenden beiden Kräfle irgend eine Kraft-Einheit angenommen, so läfst. sich, da auch die LängenEinheit für die Linien willkürlich ist, offenbar eine solche Längen-Einheit annehmen, dafs die Linie $\boldsymbol{C B}=\boldsymbol{b}$ durch dieselbe Zahl $\boldsymbol{p}$ ausgedrückt wird, durch welche man die Kraft $p$ gemessen hat, so dafs also die Zahl $b$ der Zahl $\boldsymbol{p}$ gleich ist. Dann aber mufs hier, verinöge des Satzes vom Hebel (1.), wegen $b=p$, auch nothwendig $a=q$ sein; denn $a p=b q$ giebt für $b=p$, $a p=p q$, folglich $a=q$.

So drücken nun die Zahlen $\boldsymbol{p}$ und $q$ vollständig die Gröfse der beiden Kräfte und zugleich die Länge der Linien $B C$ und $A C$ aus, und die Figur giebt ihre Richtung an.

D. Man lege jetzt in der Richtung der geraden Linie $A C B$ an $A$ die Kraft $\boldsymbol{A} A_{1}=p$ und an $B$ die gleiche Kraft $\boldsymbol{B B} B_{1}=p$, so ändern diese neu hinzukommenden Kräfte an der Wirkung auf den Hebel nichts; denn sie heben sich auf.

E. Statt der beiden gleichen Kräfte $\boldsymbol{A} A_{1}=p$ und $\boldsymbol{A} A_{2}=p$ setze man eine mittlere Kraft $\boldsymbol{A A}_{3}$, die eben so auf den Punct $\boldsymbol{A}$ wirkt, wie jene beiden Kräfte. Die Richtung dieser mittlern Kraft macht nothwendig mit $\boldsymbol{A}_{1}$ und $\boldsymbol{A A}_{2}$ gleich gro/se Winkel $\boldsymbol{A}_{1} \boldsymbol{A} \boldsymbol{A}_{3}=\boldsymbol{A}_{2} \boldsymbol{A} \boldsymbol{A}_{3}$. Denn da die Kräfte $\boldsymbol{A} A_{1}$ und $\boldsymbol{A} A_{2}$ gleich gro/s sind, so ist kein Grund vorhanden, weshalb die Richtung der mittlern Kraft derjenigen der einen Kraft näher liegen sollte, als der der andern. Die Grö/se der mittlern Kraft bleibe einstweilen dahingestellt.

F. Eben so setze man statt der beiden Kräfte $B B_{1}=p$, und $B B_{2}=q$ eine mittlere Kraft, die eben so auf den Punct $B$ wirkt, wie jene beiden. Von dieser mittlern Kraft sind sowohl Richtung als Grö/se noch unbekannt. Von der Richtung weifs man blofs, dafs sie, wie z. B. die Linie $\boldsymbol{B B}_{3}$, zwischen die Linien $\boldsymbol{B} B_{1}$ und $\boldsymbol{B} B_{2}$ fallen mufs; denn sie kann weder in eine dieser Linien, noch au/serhalb des Winkels $\boldsymbol{B}_{1} \boldsymbol{B} \boldsymbol{B}_{2}$ fallen.

G. Da aber auf diese Weise die Winkel $A_{3} A B$ und $B_{3} B A$ zusammen jedenfalls gröfser als zwei rechte sind, indem $A_{2} A B$ und $B_{2} B A$ zusammen zwei rechte ausmachen, so müssen sich $\boldsymbol{A}_{3} \boldsymbol{A}$ und $\boldsymbol{B}_{3} \boldsymbol{B}$ nothwendig oberhalb $\boldsymbol{A B}$ irgendwo schneiden. Es geschehe in $\boldsymbol{M}$; von wo nun auch eine Linie $\boldsymbol{M M _ { 3 }}$, mit $\boldsymbol{A} \boldsymbol{A}_{2}$ und $\boldsymbol{B B _ { 2 }}$ parallel gezogen, die Linie $\boldsymbol{A B}$ zwischen $\boldsymbol{A}$ und $B$ treffen mufs.

H. Nun stelle man sich $\boldsymbol{A} \boldsymbol{M}$ und $\boldsymbol{B} \boldsymbol{M}$ etwa als feste Stangen vor. Legt man dann an den Punct $\boldsymbol{M}$ dieselben vier Kräfte, welche an $\boldsymbol{A}$ und $\boldsymbol{B}$ 
wirken, nach denselben Richtungen, nemlich die Kraft $\boldsymbol{M M}_{1}=p=\boldsymbol{A A}_{1}$ parallel mit $\boldsymbol{A A}$, die Kraft $\boldsymbol{M M _ { 2 }}=\boldsymbol{p}=\boldsymbol{B} B_{1}$, parallel mit $\boldsymbol{B B _ { 1 }}$, und die Kräfte $M M_{3}=p=\boldsymbol{A A}_{2}$ und $\boldsymbol{M M _ { 4 }}=q=\boldsymbol{B B}_{2}$ parallel mit $\boldsymbol{p}$ und $q$, so werden diese vier Kräfte $\boldsymbol{M M _ { 1 }}, \boldsymbol{M M}_{2}, \boldsymbol{M M}_{3}$ und $\boldsymbol{M M _ { 4 }}$ eben so auf den Hebel $\boldsymbol{A B}$ wirken, wie die vier Kräfte $\boldsymbol{A A}_{1}, \boldsymbol{A A}_{2}, \boldsymbol{B B _ { 1 }}$ und $\boldsymbol{B} \boldsymbol{B}_{2}$, und folglich auch eben so, wie blofs die beiden gegebenen Kräfte $\boldsymbol{A A}_{2}=p$ und $\boldsymbol{B} \boldsymbol{B}_{2}=q$, indem die beiden übrigen Kräfte $\boldsymbol{A A}_{1}=p$ und $\boldsymbol{B} \boldsymbol{B}_{1}=p$ sich aufheben $(\boldsymbol{D}$.$) . Und$ da auch in $\boldsymbol{M}$ die beiden gleichen Kräfte $\boldsymbol{M} M_{1}=p$ und $M M_{2}=p$ sich aufheben, so bleiben nur die beiden Kräfte $\boldsymbol{M M}_{3}=\boldsymbol{p}$ und $\boldsymbol{M} \boldsymbol{M}_{4}=q$ übrig, die also nun eben so auf den Hebel wirken, wie die $\boldsymbol{A A}_{2}=\boldsymbol{p}$ und $\boldsymbol{B B _ { 2 }}=q$. Sie fallen in eine und dieselbe Linie, nach gleicher Richtung, und folglich machen sie zusammen die Kraft

\section{(2.) $p+q$}

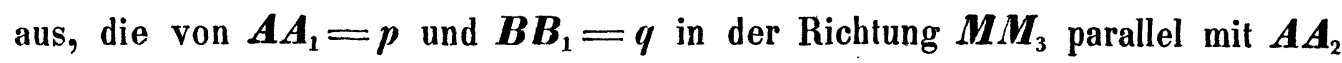
und $\boldsymbol{B} \boldsymbol{B}_{2}$ hervorgebracht wird. Deshalb wird dann auch, wenn man in demjenigen 'Punct, in welchem $\boldsymbol{M M}_{3}$ die $\boldsymbol{A B}$ schneidet, eine Kraft $\boldsymbol{p}+\boldsymbol{q}$ in entgegengesetzter Richtung $M_{3} M$ an den Hebel anbringt, diese Kraft den Kräften $\boldsymbol{A} \boldsymbol{A}_{2}=\boldsymbol{p}$ und $\boldsymbol{B} \boldsymbol{B}_{2}=q$ das Gleichyewicht halten und folglich jener Schneidepunct der Ruhepunct des Hebels sein.

I. Da die für die mittlere Kraft von $B B_{1}=p$ und $B B_{2}=q$ vorausgesetzte Richtung $\boldsymbol{B B}_{3}$ noch unbekannt ist, so ist auch der Punct $M$ unbekannt, wo $\boldsymbol{B}_{3} B$ verlängert, die Verlängerung der ihrer Richtung nach bekannten mittlern Kraft $A_{3} A$ schneidet, mithin auch der Schneidepunct von $\boldsymbol{M} M_{3}$ und $\boldsymbol{A B}$, und folglich auch der Ruhepunct des Hebels.

Aber der Punct $\boldsymbol{M}$ ergiebt sich aus dem als bewiesen vorausgesetzten Satz vom Hebel. Nach diesem Satze liegt der Ruhepunct $\boldsymbol{C}$ um $q$ von $\boldsymbol{A}$ und um $p$ von $B$ entfernt. Also folgt umgekehrt, dafs der Punct $M$, in welchem sich die Richtungen $A_{3} A$ und $B_{3} B$ der mittleren Kräfle von $A A_{1}=p$, $\boldsymbol{A A}_{2}=p$ und von $B B_{1}=p$ und $B B_{2}=q$ verlängert schneiden, derselbe ist, in welchem eine mit $\boldsymbol{A A}_{2}$ oder $\boldsymbol{B B}_{2}$ durch $\boldsymbol{C}$ gézogene Parallele die Verlängerung von $A_{3} A$ trift.

Dadurch ist der Punct $M$ vollständig bestimmt. Und da die Winkel $C A M, A_{1} A A_{3}, A_{2} A A_{3}$ und $M A C$ einander gleich sind, so ist das Dreieck $A M C$ über $A M$ gleichschenktig, und folglich ist $M C=A C=q$ und $M_{4}$ fällt in $C$, wegen $M C=A C=q$. 
15. Vom Parallelogramm- und dem Parallelepipedum der Kräfte.

K. Da jetzt der Punct $\boldsymbol{M}$ bekannt ist, so ist nun auch die Richtung $\boldsymbol{M B}$ der Verlängerung der vorausgesetzten Richtung $\boldsymbol{B B}_{3}$ der mittleren Kraft von $\boldsymbol{B} B_{1}=p$ und $\boldsymbol{B} B_{2}=q$ bekanut. Sie ist die der Diagonal des Vierecks $\boldsymbol{M M}_{2} \boldsymbol{C B}$; und dieses Viereck ist ein Parallelogramm, weil $\boldsymbol{M} \boldsymbol{M}_{2}=\boldsymbol{C B}$ $=\boldsymbol{p}$ und parallel mit $\boldsymbol{C B}$ ist. Dem Parallelogramm $\boldsymbol{M}_{t} \boldsymbol{M}_{2}$ ist aber, wenn man $\boldsymbol{B}_{2} \boldsymbol{B}_{3}$ mit $\boldsymbol{B} B_{1}$ und $\boldsymbol{B}_{1} \boldsymbol{B}_{3}$ parallel mit $\boldsymbol{B} B_{2}$ zieht, die sich dann in der verlängerten $M B$ schneiden, das Parallelogramm $\boldsymbol{B}_{2} \boldsymbol{B}_{1}$ gleich: also ist die Richtung der mittleren Kraft von $\boldsymbol{B} B_{1}=\boldsymbol{p}$ und $\boldsymbol{B} \boldsymbol{B}_{2}=q$ die der Diagonal des über $\boldsymbol{p}$ und $q$ beschriebenen Parallelogramms. So ist die Richtung der mittleren Kraft von $\boldsymbol{p}$ und $q$ gefunden.

L. Es kommt nun noch auf die Grö/se der mittlern Kraft von $p$ und $q$ an, die durch $z$ bezeichnet werden mag.

Man setze, es sei $z$ nicht gleich der Diagonal $\boldsymbol{B B}_{3}$ des Parallelogramms $\boldsymbol{B}_{1} \boldsymbol{B}_{2}$, sondern z. B. kleiner, und gleich $\boldsymbol{B N}$. Dann müfste eine Kraft $z=B N_{1}=B N$, in entgegengesetzter Richtung von $B N$, mit den beiden Kräften $\boldsymbol{B} B_{1}=p$ und $\boldsymbol{B B}_{2}=q$ im Gleichgewicht sein. Also müfste auch die mittlere Kraft der beiden Kräfte $B N_{1}=z$ und $B B_{2}=q$ grade die entgegengesetzte Richtung der dritten Kraft $\boldsymbol{B B}_{1}=\boldsymbol{p}$ haben. Aber die mittlere von zwei Kräften hat immer, wie oben gefunden, die Richtung der Diagonal des über den beiden Kräften beschriebenen Parallelogramms. Also würde hier die mittlere Kraft von $B N_{1}=z$ und $B B_{2}=q$ die Richtung der Diagonal $B N_{2}$ des Parallelogramms $B N_{1} N_{2} B_{2}$ haben. Diese Richtung ist aber offenbar nicht die Verlängerung von $\boldsymbol{B} B_{1}$. Also kạn nicht $z<\boldsymbol{B B}_{3}$ sein.

Setzt man $z>B B_{3}$, z. B. $=B L$ und $B L_{1}=B L$, so müfste die mittlere Kraft von $B L_{1}=z$ und $B B_{2}=q$ die Richtung der Diagonal $B L_{2}$ des über $B L_{1}$ und $B B_{2}$ beschriebenen Parallelogramms $B L_{1} L_{2} B_{2}$ haben. Diese Richtung ist aber wiederum nicht die Verlängerung von $B B_{1}$. Mithin kann auch nicht $\approx>B B_{3}$ sein.

Folglich kann nur $\approx$ gleich $\boldsymbol{B B}_{3}$ sein, und die Diagonal $\boldsymbol{B B}_{3}$ des über $\boldsymbol{B B}_{1}=p$ und $\boldsymbol{B B}_{2}=q$ beschriebenen Parallelogramms $\boldsymbol{B B}_{1} \boldsymbol{B}_{2} \boldsymbol{B}_{3}$. drückt die mittlere Kraft von $\boldsymbol{p}$ und $q$ sowohl nach Grö/se als nach Richtung aus; welches der Satz vom Parallelogramm der Kräfte ist, begründet auf dem Satz vom Hebel. 
15. Vom Parallelogramm- und dem Parallelepipedum der Krüfte. 225

Beweis des Satzes vom Hebcl, wenn der Satz vom Parallelogramm der Kräfte als bewiesen vorausgesetzt wird.

3.

A. Es ist zu diesem Beweise nicht der vollständige Satz vom Parallelogramm der Kräfte, sondern nur der Satz (\$. 2. K.) nöthig, nemlich, dafs die mittlere Kraft zweier Kräfte $p$ und $q$ die Richlung der Diagonal des über $p=q$ beschriebenen Parallelogramms hat.

B. Man lege nemlich, wie oben, an den Hebel noch die beiden sich aufhebenden Kräfte $\boldsymbol{A} \boldsymbol{A}_{1}=\boldsymbol{p}$ und $\boldsymbol{B B _ { 1 }}=p$, so kommt es nur darauf an, in welchem Puncte $M$ die Richtungen $A_{3} A M$ und $\boldsymbol{B}_{3} B M$ der mittleren Kräfte von $\boldsymbol{A} A_{1}=p, A A_{2}=q$ und von $B B_{1}=p$ und $B B_{2}=q$ sich schneiden. Denn legt man an diesen Punct $\boldsymbol{M}$, wie oben, die vier Kräfte $\boldsymbol{M} \boldsymbol{M}_{1}=\boldsymbol{p}$, $\boldsymbol{M} \boldsymbol{M}_{2}=\boldsymbol{p}$ parallel mit $\boldsymbol{A B}$, und $\boldsymbol{M} \boldsymbol{M}_{3}=\boldsymbol{p}$ und $\boldsymbol{M} \boldsymbol{M}_{4}=q$ parallel mit $\boldsymbol{A} \boldsymbol{A}_{2}$ oder $\boldsymbol{B} B_{2}$, so wirken dieselben eben so auf den Hebel, wie die beiden gegebenen Kräfte $\boldsymbol{p}$ und $q$, und der Punct $\boldsymbol{C}$, in welchem eine Parallele mit $\boldsymbol{A} \boldsymbol{A}_{2}$ oder $\boldsymbol{B B}_{2}$, durch $\boldsymbol{M}$ gezogen, die Linie $\boldsymbol{A B}$ schneidet, ist der Ruhepunct des Hebels, in welchem eine Kraft $\boldsymbol{M} \boldsymbol{M}_{3}+\boldsymbol{M} \boldsymbol{M}_{4}=\boldsymbol{p}+q$, in entgegengesetzter Richtung von $p$ und $q$ angebracht, den Kräften $p$ und $q$ das Gleichgewicht hält.

$C$. Ist nun bewiesen, dafs die mittleren Kräfte von $B B_{1}=p$ und $B B_{2}=q$ und von $A A_{1}=p$ und $A A_{2}=p$ die Richtungen $B B_{3}$ und $A A_{3}$ der Diagonalen der Parallelogramme $B_{1} B_{2}$ und $A_{1} A_{2}$ haben, so werden die Richtungen der Linien $\boldsymbol{A} \boldsymbol{M}$ und $\boldsymbol{B} \boldsymbol{M}$ durch die Winkel $\alpha$ und $\beta$ in den Dreiecken $\boldsymbol{A C M}$ und $\boldsymbol{B C M}$ bestimmt. Zieht man daher $\boldsymbol{M C}$ durch $\boldsymbol{M}$ mit $\boldsymbol{A A _ { 2 }}$ oder $\boldsymbol{B B}_{2}$ parallel, so mufs

(3.) $\frac{B C}{M C}=\frac{B B_{1}}{B_{1} B_{2}}=\frac{p}{q}$ und $\frac{A C}{M C}=\frac{A A_{1}}{A A_{2}}=\frac{p}{q}=1$

sein. Dies giebt, Eins durch das Andere dividirt:

und dies ist der Satz vom Hebel.

$$
\text { (4.) } \frac{B C}{A C}=\frac{p}{q} \text {; }
$$

Vorbemerkungen zu andern unabhängigen Beweisen der Sätze vom Parallelogramm der Kräfte und vom Hebel.

4.

A. Eine $\boldsymbol{K r}$ raft wird durch die Zahl, welche angiebt, wievielmal sie eine zur Einheit' angenommene Kraft enthält, und durch eine gerade Linie 
15. Vom Parallelogramm- und dem Parallelepipedum der Kräfte.

bestimmt, welche die Richtung der Kraft hat. Giebt man dieser Linie eine bestimmte Länge, welche ihrerseits durch die Zahl der in der Länge enthaltenen Längen-Einheiten gemessen wird, so läfst sich, wenn man die Zahl der Längen-Einheiten der Zahl der in der Kraft enthaltenen Kraft-Einheiten entweder gleich setzt, oder beide in ein bestimmtes Verhältnifs bringt, die Kraft durch die Linie, nach Richtung und Stärke vollständig bildlich darstellen. Also wird eine Kraft ,überhaupt durch Zahl und Form, oder durch Zahl und Raum dargestellt.

B. Dies reicht in den Fällen, wo Kräfte einander das Gleichgeuicht halten, und wo folglich keine Beuregung entsteht, also in der Statik, völlig aus; wie es sich z. B. beim Parallelogramm der Kräfte zeigt. Aber wenn Kräfte nicht einander das Gleichgewicht halten, sondern Bewegung erfolgt, reicht die obige bildliche Darstellung der Kräfte nicht hin, weil die Bewegung durch Richtung und Ausdehnung, also durch Zahl und Raum noch nicht vollständig gemessen wird, sondern auch noch die Zeit in Betracht kommt; denn es kommt darauf an, in welcher Zeit diese oder jene Ausdehnung in dieser oder jener Richtung durchlaufen wird. Die Zeit wird ihrerseits durch eine Zahl von willkürlich angenommenen Zeit-Einheiten gemessen. Also sind zum Messen der Bewegung zusammen Zahl, Richtung oder Raum, und Zeit nöthig.

Die Zahl oder Menge ist ein Urbegriff; die Richtung oder die Form des Raums ist es gleichfalls, und die Zeit ebenfalls. Alle drei (nicht blofs die beiden: Raum und Zeit) sind für die Gröfsenlehre, wenn man es so nennen will, Formen der Wahrnehmung.

Die Analysis oder Rechenkunst beschäftigt sich blofs mit der Zahl, ohne weitere Anwendung auf die Dinge. Die Geometrie verbindet bei ihren Untersuchungen Zahl und Raum; die Mechanik: Zahl, Raum und Zeit. Rechenkunst, Geometrie und Mechanik sind auf diese Weise die drei wesentlichen Theile der Mathematik oder Gröfsenlehre.

C. Für denjenigen Theil der Mechanik, welcher blofs das Gleichgewicht der Kräfte, nicht die Bewegung betrachtet, ist der Begriff der Zeit gleichgültig; sie kann, von Null bis Unendlich, sein was man will. Die Statik stellt, wie schon bemerkt, die Stärke der Kräfte durch die:Länge von geraden Linien bildlich, und die Richtungen der Kräfte durch die Richtung dieser Linien vor, und bedarf so nur der Urbegriffe von Zahl und Raum.

D. Aber es hindert nichts, das Gleichgewicht von Kräften. auch auf die Weise zu untersuchen, dafs man annimmt, diese oder jene, zugleich wir- 
kenden Kräfte kämen neben einander wirklich zu ihrer Wirkung und erzeugten Bewegung. Ist dann das End-Ergebnifs dasselbe, wie das von der Wirkung dieser oder jener andern zugleich wirkenden, ebenfalls neben einander zur Wirkung gelangenden Kräften, so darf man mit Grunde schliefsen, dafs die erste Gruppe von Kräften, sugleich wirkend, der zweiten Gruppe (die letzte in grade entgegengesetzten Richtungen angenommen) das Gleichgewicht halten werde, weil die zweite Gruppe, nach der ersten und in entgegengesetzten Richtungen wirkend, die Masse, auf welche die Wirkung geschieht, grade wieder nach demselben Orle zurückführen würde, von welchem die erste Gruppe sie entfernte.

Diese Ansicht wollen wir auf den vorliegenden Gegenstand anzuwenden versuchen. Es werden dazu folgende Lehnsätze nöthig sein.

L e h n s ä t z e.

5.

A. Wirkungen verhalten sich unter sonst gleichen Umständen wie die Ursachen; und umgekehrt. Wenn man will, kann man diesem Satze auch noch erst den einfacheren Satz vorausschicken, dafs gleiche Ursachen gleiche Wirkungen haben, und umgekehrt; was offenbar ohne Beweis richtig und folglich Grundsatz ist; und dann den Satz erst für rationale Verhältnisse von Ursachen und Wirkungen, hierauf, nach der gewöhnlichen Art des Überganges vom Rationalen zum Irrationalen, auch für irrationale Verhältnisse beweisen.

B. Die Geschwindigkeiten $c$ und $\boldsymbol{C}$, welche eine auf eine und dieselbe träge Masse $\boldsymbol{M}$ unveränderlich stark fortwirkende, sich gleich bleibende Kraft $\boldsymbol{P}$ also auch die sich gleichbleibende beschleunigende $\boldsymbol{K r a f t} \boldsymbol{p}=\frac{\boldsymbol{P}}{\boldsymbol{M}}$ (nemlich der auf die Einheit der Masse kommende Theil der Kraft) in verschiedenen Zeiträumen $\boldsymbol{t}$ und $\boldsymbol{T}$ hervorbringt, verhalten sich wie diese Zeiträume, und umgekehrt, so dafs

$$
\text { (5.) } \quad \frac{C}{c}=\frac{T}{t} \text { und } \frac{C}{T}=\frac{c}{t} \text { ist. }
$$

Denn die verschiedene Dauer der sich gleichbleibenden Kraft und die Masse sind hier die Ursachen und die hervorgebrachten Geschwindigkeiten die Wirkungen, und diese verhalten sich wie jene $(\boldsymbol{E}$.$) .$

$C$. Die Geschwindigkeiten $c$ und $C$, welche verschiedene, auf eine 
228 15. Vom Parallelogramm- und dem Parallelepipedım der Kräfte.

und dieselbe träge Masse $M$ unveränderlich stark fortwirkende, sich gleich bleibende Kräfte $\boldsymbol{Q}$ und $\boldsymbol{P}$, also auch die sich gleichbleibenden beschleunigenden Kräfte $p=\frac{P}{M}$ und $q=\frac{Q}{M}$ in gleichen Zeittheilen $t$ hervorbringen, verhalten sich wie die Kräfle, und umgekehrt, so dafs

$$
\text { (6.) } \frac{c}{C}=\frac{Q}{P}=\frac{q}{p} \text { ist. }
$$

Denn die verschiedenen, für dieselbe Masse und Zeit sich gleichbleibenden Kräfte sind hier die Ursachen: die in den gleichen Zeittheilen hervorgebrachten Geschwindigkeiten sind wieder die Wirkungen; und diese verhalten sich wie jene (A.).

$\boldsymbol{D}$. Die $\boldsymbol{R a ̈ u m e} \boldsymbol{r}$ und $\boldsymbol{R}$, durch welche eine und dieselbe Masse $\boldsymbol{M}$ von zwei verschiedenen, unveränderlich stark fortwirkenden bewegenden Kräften $\boldsymbol{Q}$ und $\boldsymbol{P}$, deren zugehörige beschleunigende Kräfte $q$ und $\boldsymbol{p}$ also $\boldsymbol{q}=\frac{\boldsymbol{Q}}{\boldsymbol{M}}$ und $\boldsymbol{p}=\frac{\boldsymbol{P}}{\boldsymbol{M}}$ sind, in gleichen Zeittheilen $\boldsymbol{T}$ fortgetrieben wird, verhalten sich wie die Kräfte, und umgekehrt; so dafs also

$$
\text { (7.) } \frac{R}{r}=\frac{P}{Q}=\frac{p}{q} \text { ist. }
$$

a. Denn man stelle, bildlich, durch die Abscissen $A t_{1}, A t_{2}, \ldots A T$ (Fig. 2) verschiedene Zeiträume $t_{1}, t_{2}, \ldots T$, und durch die auf diese Abscissen senkrechten Ordinaten $t_{1} c_{1}, t_{2} c_{2}, \ldots T c$ und $t_{1} C_{1}, t_{2} C_{2}, \ldots T C$ die von zwei verschiedenen bewegenden Kräften $\boldsymbol{Q}$ und $\boldsymbol{P}$ auf eine und dieselbe Masse $\boldsymbol{M}$, also die von den beschleunigenden Kräften $q=\frac{Q}{M}$ und $p=\frac{P}{M}$ in den Zeiträumen $t_{1}, t_{2}, \ldots T$ hervorgebrachten Geschwindigkeiten $c_{1}, c_{2}, \ldots c$ und $\boldsymbol{C}_{1}, \boldsymbol{C}_{2}, \ldots \boldsymbol{C}$ vor, so bezeichnen offenbar, eben so bildlich, die Flächen $f$ und $\boldsymbol{F}$ der beiden Figuren $\boldsymbol{T A}_{1} c_{2} \ldots c$ und $\boldsymbol{T A C}_{1} \boldsymbol{C}_{2} \ldots C$ die Räume $r$ und $\boldsymbol{R}$, durch welche der Körper von den beiden beschleunigenden Kräften $q$ und $\boldsymbol{p}$ in der gleichen Zeit $\boldsymbol{T}$ fortgetrieben wird.

b. Nun ist für die Kraft $q$ nach (5. B.) $\frac{c_{1}}{t_{1}}=\frac{c_{2}}{t_{2}} \cdots=\frac{c}{T}$ und für die Kraft $p, \frac{C_{1}}{t_{1}}=\frac{C_{2}}{t_{2}} \cdots=\frac{C}{T}$, also ist in der Figur:

$$
\text { (8.) } \quad \frac{c_{1} t_{1}}{A t_{1}}=\frac{c_{2} t_{2}}{A t_{2}} \cdots=\frac{c T}{A T} \text { und } \frac{C_{1} t_{1}}{A t_{1}}=\frac{C_{2} t_{2}}{A t_{2}} \cdots=\frac{C T}{A T} \text {, }
$$

und folglich sind $A c_{1} c_{2} \ldots c$ und $A C_{1} C_{2} \ldots C$ gerade Linien und die Figuren $\boldsymbol{T A C}_{1} \boldsymbol{c}_{2} \ldots c$ und $\boldsymbol{T A C} \boldsymbol{C}_{1} \ldots \boldsymbol{C}$ sind geradlinige rechtwinklige Dreiecke. 
15. Vom Parallelogramm- und dem Parallelepipedum der Kräfte.

c. Die Flächen $\boldsymbol{f}$ und $\boldsymbol{F}$ dieser Dreiecke sind

(9.) $f=\frac{1}{2} A T \times T c$ und $\boldsymbol{F}=\frac{1}{2} A T \times T C$.

Und da $\boldsymbol{A T}$ die Zeit $\boldsymbol{T}$ und $\boldsymbol{T} \boldsymbol{c}$ und $\boldsymbol{T} \boldsymbol{C}$ die Geschwindigkeiten $c$ und $\boldsymbol{C}$ vorstellen, so ist

(10.) $f=\frac{1}{2} \boldsymbol{T} \boldsymbol{c}$ und $\boldsymbol{F}=\frac{1}{2} \boldsymbol{T C}$.

$d$. Die Flächen $f$ und $F$ stellen aber die Räume vor, durch welche die Kräfte $\boldsymbol{p}$ und $\boldsymbol{Q}$ den Körper in der gleichen Zeit $\boldsymbol{T}$ getrieben haben $(a)$; also ist$$
\text { (11.) } r=\frac{1}{2} T c \text { und } R=\frac{1}{2} T C \text {. }
$$

e. Hieraus folgt

$$
\text { (12.) } \frac{r}{R}=\frac{c}{C} \text {, }
$$

und da zufolge (6.C.) $\frac{c}{C}=\frac{Q}{P}=\frac{q}{p}$ ist:

wie (7.).

$$
\text { (13.) } \frac{\boldsymbol{R}}{\boldsymbol{r}}=\frac{\boldsymbol{P}}{\boldsymbol{Q}}=\frac{p}{q},
$$

$\boldsymbol{E}$. Wenn die bewegende Kraft $\boldsymbol{S}$ die Masse $\boldsymbol{M}$ in der Zeit $\boldsymbol{T}$ durch den Raum $r$ zu treiben vermag, so vermag sie die Masse $m$ in derselben Zeit $\boldsymbol{T}$ durch den Raum $\boldsymbol{R}=\frac{M r}{m}$ zu treiben, so dafs

$$
\text { (14.) } M r=m R \text { ist. }
$$

Denn die beschleunigenden $K r a ̈ f t e$, welche $S$ auf $M$ und $m$ hervorbringt, seien

$$
\text { (15.) } q=\frac{S}{M} \text { und } p=\frac{s}{m} \text {. }
$$

Diese beschleunigenden Kräfte wirken auf gleiche Massen, nämlich auf die gemeinschaftliche Einheit der Massen $M$ und $m$; also ist nach (13.)

$$
\text { (16.) } \frac{\boldsymbol{R}}{r}=\frac{p}{q}
$$

und, hierin die Ausdrücke von $p$ und $q$ (15.) gesetzt:

wie (14.)

$$
\text { (17.) } \frac{r}{R}=\frac{S}{M}: \frac{S}{m}=\frac{m}{M} \text { oder } M r=m R \text {, }
$$

Unabhängiger Beweis des Satzes vom Parallelogramm der Kräfte; ohne den Satz vom Hebel.

6.

A. Wenn auf eine Masse in $C$ (Fig. 3) zwei gleiche Kräfte $D_{1} C=r$ und $D_{2} C=r$ zugleich wirken, so wird es immer eine inittlere Kraft $A C=p$ 
geben, welche auf $\boldsymbol{C}$ dieselbe Wirkung hervorbringt, wie die beiden gleichen Kräfte $r$. Ihre Richtung $A C$ wird den Winkel $D_{1} C D_{2}$ halbiren; ihre Gröfse oder Stärke bleibt einstweilen dahingestellt.

Wenn nemlich die beiden Kräfte $\boldsymbol{r}$ auf $\boldsymbol{C}$ zugleich wirken, so kann $\boldsymbol{C}$ offenbar weder in der Richtung $D_{1} C D_{4}$, noch in der Richtung $D_{2} C D_{3}$ fortgetrieben werden. Die Kraft $\boldsymbol{D}_{2} C$ lenkt $C$ von der Richtung $D_{1} C_{4}$ nach oben hin, und die Kraft $D_{1} C$ von der Richtung $D_{2} C D_{3}$ nach unlen hin ab, so dafs sich $\boldsymbol{C}$ in einer Richtung $\boldsymbol{A C E}$ fortbewegen wird, welche zwischen $D_{1} C$ und $D_{2} C$ fällt. Aber es ist kein Grund vorhanden, warum $A C$ näher an $D C_{1}$ als an $D_{2} C$ liegen sollte, und folglich wird $A C$ den Winkel $D_{1} C D_{2}$ halbiren. Und da nun $C$ wirklich in dieser Richtung $A C$ sich fortbewegen mufs, so wird es auch nothwendig irgend eine nach $A C$ selbst wirkende Kraft $\boldsymbol{p}$ geben, welche $\boldsymbol{C}$ eben so forttreibt, wie die beiden zugleich wirkenden gleichen Kräfte $D_{1} C=r$ und $D_{2} C=r$.

$\boldsymbol{B}$. Wenn auf eine, von einer bewegenden Kraft $\boldsymbol{P}$, oder von einer beschleunigenden Kraft $\boldsymbol{p}=\boldsymbol{C A}$ (Fig. 4) in der Richtung $\boldsymbol{A C}$ fortgetriebene Masse in $C$, eine zweite beschleunigende Kraft $q=B C$ in der auf $A C$ senkrechten Richtung $B C$ wirkt, so behält $\dot{p}$ in allen mit $A C$ parallelen Richtungen ihre volle Wirkung. Sie wird in allen diesen Richtungen durch $q$ weder verstärkt noch geschwächt; $p$ wirkt in allen diesen Richtungen so, als wenn $q$ gar nicht da wäre.

Denn es sei erst blofs $\boldsymbol{p}$ ohne $q$ vorhanden und man lasse auf $\boldsymbol{C}$ noch zwei andere gleiche Kräfte $D_{1} C=r$ und $D_{2} C=r$ in Richtungen wirken, die mit $A C$ gleiche Winkel machen. Dann wird die Richtung der mittleren Kraft, welche zufolge (A.) die Stelle der beiden Kräfte $r$ vertritt in $\boldsymbol{A C}$ fallen. Jede der beiden gleichen Kräfte $r$ aber wird zu der mittleren Kraft offenbar einen gleichen Theil $s$ beitragen: also wird $p$ durch die beiden gleichen Kräfte $r$ um die Kraft $2 s$ verändert werden. Nun lasse man den beiden gleichen Kräften $D_{1} C=r$ und $D_{2} C=r$ zwei andere gleiche Kräfte $D_{3} C=r$ und $\boldsymbol{D}_{4} \boldsymbol{C}=\boldsymbol{r}$ grade entgegen wirken: so wird auch die die Stelle dieser gleichen Kräfte $\boldsymbol{D}_{3} C=\boldsymbol{r}$ und $\boldsymbol{D}_{4} C=\boldsymbol{r}$ vertretende mittlere Kraft die Kraft $\boldsymbol{p}$ um $2 \sigma$ verändern und ihre Richtung wird in die gerade Linie $E C A$ fallen. Aber die Kräfte $D_{1} C=r$ und $D_{4} C=r$, so wie $D_{2} C=r$ und $D_{3} C=r$, heben sich gegenseitig auf. Also müssen auch ihre mitlleren Kräfte $2 s$ und $2 \sigma$ sich aufheben, so dafs $s=\sigma$ sein mufs; und die Wirkung auf $C$ wird so sein, als wenn die vier Kräfte $r$ gar nicht da wären. Aber, da $s=\sigma$ 
sein mufs, so werden auch die Theile $s$ und $\sigma$, welche die Kräfte $D_{2} C=r$ und $D_{4} C=r$ zu der Wirkung, auf $C$ in der Richtung $A C$ beitragen, einzeln sich aufheben, und diese beiden Kräfte werden auf $C$ in der Richtung $A C E$ gar keine Wirkung haben. Die Stelle der beiden Kräfte $D_{2} C=r$ und $D_{4} C$ kann nun zufolge $(A$.$) eine mittlere \mathrm{Kraft}$, welche $=q$ sein mag, vertreten, die dann Dasselbe thut, wie jene beiden. Die Richtung $B C$ dieser mittleren Kraft $q$ wird zufolge ( $A$.) den Winkel $\boldsymbol{D}_{2} C D_{4}$ halbiren, also auf $A C E$ senkrecht stehen. Mithin hat die nach $B C$ senkrecht auf die Richtung $A C$ von $p$ wirkende Kraft $q$ auf die Bewegung der Masse in $\boldsymbol{C}$ nach $\boldsymbol{A C}$, und nach allen Richtungen die mit $A C$ parallel sind, gar keine Wirkung.

$C$. Wenn zwei beschleunigende Kräfte $p$ und $q$, deren Richtungen $\boldsymbol{A C}$ und $\boldsymbol{B C}$ (Fig. 5) auf einander senkrecht stehen und deren Stärke durch die Linie $\boldsymbol{A C}$ und $\boldsymbol{B C}$ vorgestellt wird, gleichseitig auf $\boldsymbol{C}$ wirken, so wird eine mittlere Kraft $s$, deren Richtung und Stärke durch die Richtung und Länge der Diagonal $D c$ des Rechtecks $\boldsymbol{A B C D}$ vorgestellt wird, auf $\boldsymbol{C}$ dieselbe Wirkung haben, wie $p$ und $q$ zusammen.

a. Denn man setze, die Kraft $\boldsymbol{p}$ könne die Masse in $\boldsymbol{C}$ in einer Ebene, in welcher $\boldsymbol{A C B}$ liegt, frei in der Richtung $\boldsymbol{A C}$, so wie in jeder damit parallelen Richtung, forttreiben, und sie treibe sie wirklich in der Zeit $\boldsymbol{T}$ durch die Länge $\boldsymbol{C A}_{1}=\boldsymbol{R}$ fort. Ferner stelle man sich vor, $\boldsymbol{q}$ könne $\boldsymbol{C}$, nur zusammen mit der Ebene $A C B$ selbst, in der Richtung $B C$, so wie in allen damit parallelen Richtungen, frei forttreiben und sie treibe so die Masse in derselben Zeit $\boldsymbol{T}$ durch die Länge $\boldsymbol{C B}=\boldsymbol{r}$ fort. Dann gelangt $\boldsymbol{C}$ durch die gleichzeitige Wirkung von $p$ und $q$ nach einem Puncte $D_{1}$ in der verlängerten Ebene durch $\boldsymbol{A C B}$, der von der Linie $B C B_{1}$ um die Länge $C_{1}=R$ und von der Linie $A C A$, um die Länge $C B_{1}=\dot{r}$ senkrecht entfernt ist. Denn während die Masse in $C$ durch $p$ von $C$ bis $A_{1}$ fortgetrieben wurde, hat $q$ sie, sammt der Ebene, um $C B_{1}=r$ von der Linie $A C A_{1}$ senkrecht entfernt, so dafs die Linie $\boldsymbol{A C A _ { 1 }}$ in die um $\boldsymbol{r}$ von ihr entfernte Parallele $E B E_{1}$ und also $C$ nach einem Puncte $D_{1}$ gelangt ist, der um $R$ von $B C B_{1}$ und um $r$ von $A C A_{1}$ senkrecht absteht. Dieser Punct $D_{1}$ ist der $C$ gegenüberliegende Eckpunct des Rechtecks $\boldsymbol{A}_{1} \boldsymbol{B}_{1}$ unter $\boldsymbol{R}$ und $\boldsymbol{r}$.

b. Bei der gleichzeitigen Wirkung von $p$ und $q$ ist die Richtung von $\boldsymbol{r}$ immer mit $\boldsymbol{A C}$ und die Richtung von $q$ immer mit $\boldsymbol{B C}$ parallel, also immer senkrecht auf $\boldsymbol{A C}$ geblieben. Nun haben zufolge $(B$.) zwei Kräfte, deren Richtungen auf einander senkrechl stehen, jede auf die andere, in dieser 
232 15. Vom Parallelogramm- und dem Parallelepipedum der Kräfte.

ihrer Richtung gar keinen Einflufs: also ist die Masse in $\boldsymbol{C}$ von $\boldsymbol{p}$ frei und ganz so fortgetrieben worden, als wenn $q$ gar nicht da wäre, und von $q$ ganz so, als wenn $p$ gar nicht da wäre. Und da also auf diese Weise die Kräfte $p$ und $y$ ihre volle Wirkung hatten, so verhalten sich die Räume $\boldsymbol{R}$ und $\boldsymbol{r}$, durch welche sie eine und dieselbe Masse in der Zeit $\boldsymbol{T}$ fortgetrieben haben, zufolge (\$. 5. D.), wie die Kräfle selbst und folglich ist

$$
\text { (18.) } \frac{R}{r}=\frac{B_{1} D_{1}}{A_{1} D_{1}}=\frac{p}{q}=\frac{A C}{B C} \text {. }
$$

Mithin ist $\boldsymbol{A}_{1} \boldsymbol{B}_{1}$ ein dem Reckteck $\boldsymbol{A B}$ ähnliches Rechteck.

c. Da auch, wenn für irgend einen andern Zeitraum $t$ die durchlaufenen Räume $C a=R_{1}$ und $C b=r_{1}$ sind, eben so

ist, so ist

$$
\text { (19.) } \frac{R_{1}}{r_{1}}=\frac{b d}{a d}=\frac{p}{q}=\frac{A C}{B C}
$$

$$
\text { (20.) } \quad \frac{C a}{a d}=\frac{C A_{1}}{A_{1} D}
$$

und folglich ist, da dies für alle Puncte von $C A_{1}$ und $C B_{1}$ gilt, $C D_{1}$ eine gerade Linie, und die Masse in $C$ hat unter der Wirkung der Kräfte $p$ und $q$ die Diagonal $C D_{1}$ des dem Rechteck $A B$ ähnlichen Rechtecks $A_{1} B_{1}$ durchlaufen.

d. Soll jetzt statt der beiden Kräfte $p$ und $q$ eine mittlere Kraft $s$ Statt finden, welche dieselbe Wirkung auf $C$ hat wie $p$ und $q$, und die also die Masse in $\boldsymbol{C}$ in der Zeit $\boldsymbol{T}$ allein durch die gerade Linie $\boldsymbol{C D}_{1}$ treibt, so mufs sie zunächst die Richtung der Diagonal $C_{1}$ des Rechlecks $A_{1} B_{1}$ und folglich auch die Richtung der Diagonal des ähnlichen Rechtecks $\boldsymbol{A B}$ haben. Ihre Stärke ergiebt sich aus dem Umstande, dafs, da die Masse in $C$ von $p$ durch den Raum $\boldsymbol{R}$ und von $\boldsymbol{q}$ durch den Raum $\boldsymbol{r}$ getrieben wird, die Kraft $\boldsymbol{s}$ diese Masse durch den Raum $A=C D_{1}$ mufs zu treiben vermögen. Deshalb ist denn vermöge (13. \$. 5. D.):

(21.) $\frac{s}{p}=\frac{\Delta}{C A_{1}}$ und $\frac{s}{q}=\frac{\Delta}{C B_{1}}, \quad$ also $\quad$ (22.) $s=\frac{p \Delta}{C A_{1}}=\frac{q \Delta}{C B_{1}}$.

e. Nimmt man endlich grade eine solche Zeit $\boldsymbol{T}$ an, dafs $\boldsymbol{R}$ oder $C A_{1}=A C=p$, also auch $r$ oder $C B_{1}=B C=q$, und mithin auch $C D_{1}$ oder $A=C D$ ist, so giebt (21.)

$$
\text { (23.) } \quad s=\frac{C D \cdot p}{p}=\frac{C D \cdot q}{q}=C D \text {; }
$$


also stellt die Diagonal $\boldsymbol{C D}$ des Rechtecks $\boldsymbol{A B}$, sowohl durch ihre Richlung als durch ihre Länge, diejenige mittlere Kraft $s$ vor, welche auf eine Masse in $C$ dieselbe Wirkung hat, wie die beiden auf einander senkrechten Kräfte $p$ und $q$.

D. Wenn endlich zwei Kräfte $\boldsymbol{A C}=p$ und $\boldsymbol{B C}=q$ (Fig. 6), derèn Richtungen mit einander einen beliebigen Winkel $\boldsymbol{A C B}$ machen, gleichseitig auf eine Masse in $\boldsymbol{C}$ wirken, so stellt die Diagonal $\boldsymbol{D C}$ des Parallelogramms $\boldsymbol{A B}$, unter $\boldsymbol{A C}$ und $\boldsymbol{B C}$, sowohl nach Richtung als Länge, die Richtung und Stärke der mittlern Kraft vor, die, allein auf die Masse in $\boldsymbol{C}$ wirkend, das Nemliche thut, wie $\boldsymbol{p}$ und $q$ zusammen.

a. Denn man beschreibe das Parallelogramm $\boldsymbol{A B}$ unter $\boldsymbol{A C}=\boldsymbol{\nu}$ und $\boldsymbol{B C}=q$, ziehe die Diagonal $\boldsymbol{C D}$, fälle auf dieselbe aus $\boldsymbol{A}$ und $\boldsymbol{B}$ die Perpendikel $\boldsymbol{A E}$ und $\boldsymbol{B F}$, ziehe durch $\boldsymbol{C}$ die mit den beiden parallelen Perpendikeln parallele gerade Linie $G \dot{C H}$, und dann noch $\boldsymbol{B G}$ und $\boldsymbol{A H}$ mit $\boldsymbol{C H}$ parallel, also senkrecht auf $\boldsymbol{G C H}$ : so ist $\boldsymbol{A E}=\boldsymbol{B H}=\boldsymbol{H C}=\boldsymbol{C G}$, und $\boldsymbol{E H}$ und $F G$ sind Rechtecke.

b. Nun sind $\boldsymbol{A C}$ und $\boldsymbol{B C}$ die Diagonalen der Rechtecke $\boldsymbol{E H}$ und FG: also stellt $A C$, nach Richtung und Grö/se, vermöge (C.), eine mittlere Kraft vor, welche auf $\boldsymbol{C}$ eben so wirkt, wie es zwei Kräfte $\boldsymbol{E C}$ und $\boldsymbol{H C}$, zugleich wirkend, thun würden; und $\boldsymbol{B C}$ stell, nach Richtung und Grö/se, eine mittlere $\mathrm{Kraft}$ vor, welche auf $\boldsymbol{C}$ eben so wirkt, wie es zwei Kräfte $\boldsymbol{H C}$ und $\boldsymbol{G C}$, zugleich wirkend, thun würden. Mithin würden die vier Kräfte $\boldsymbol{E} \boldsymbol{C}$, $\boldsymbol{H C}, \boldsymbol{F C}$ und $\boldsymbol{G C}$, wenn sie vorhanden wären, eben so auf $\boldsymbol{C}$ wirken, wie die beiden Kräfte $\boldsymbol{A C}$ und $\boldsymbol{B C}$. Aber die zwei Kräfte $\boldsymbol{H C}$ und $\boldsymbol{G C}$, unter den vieren, sind einander gleich und entgegengesetzt; sie heben sich also auf und wirken auf $\boldsymbol{C}$ gar nicht. Es bleiben von den auf $\boldsymbol{C}$ eben wie $"$ und $q$ wirkenden vier Kräften nur die beiden $\boldsymbol{E C}$ und $\boldsymbol{F C}$ übrig. Beide haben die Richtung $\boldsymbol{D C}$ und machen also zusammen eine Kraft $\boldsymbol{E C}+\boldsymbol{F C}$ aus, oder, weil $\boldsymbol{F} C=\boldsymbol{D E}$ ist, eine Kraft $\boldsymbol{E C}+\boldsymbol{D E}=\boldsymbol{D C}$. Mithin stellt die Diagonal $D C$ des Parallelogramms $\boldsymbol{A B}$ unter $\boldsymbol{A C}=\boldsymbol{p}$ und $\boldsymbol{B C}=\boldsymbol{q}$, nach Richtung und Grö/se, die mittlere Kraft vor, welche eben so auf $C$ wirkt, wie die beiden Kräfte $p$ und $\boldsymbol{q}$.

Dies ist der Satz vom Parallelogramm der Kräfte. 
15. Vom Parallelogramm- und dem Parallelepipedum der Kräfte.

Unabhängiger Beweis des Satzes vom Hebel; ohne den Satz vom Parallelogramm der Kräfte.

7.

A. Man stelle sich die Rollen $\boldsymbol{A C}$ und $\boldsymbol{B C}$ (Fig. 7.) mit einander verbunden und frei um die Axe $C$ beweglich, desgleichen ohne Reibung und Masse vor. In dem Puncte $\boldsymbol{A}$ wirke nach der Richtung $\boldsymbol{A P}$, senkrecht auf die gerade Linie $\boldsymbol{A C B}$, eine bewegende $K$ raft $\boldsymbol{P}$ auf die Masse $M$, und in $\boldsymbol{B}$, nach der mit $\boldsymbol{A P}$ parallelen Richtung $\boldsymbol{B} \boldsymbol{Q}$, eine bewegende Kraft $\boldsymbol{Q}$ auf die Musse $m$; und zwar sollen $\boldsymbol{P}$ und $\boldsymbol{Q}$ von der Art sein, dafs die beschleunigenden Kräfte $p$ und $q$, welche $\boldsymbol{P}$ in $\boldsymbol{M}$ und $\boldsymbol{Q}$ in $m$ hervorbringen, einander gleich sind, also so, dafs

$$
\text { 24. } \frac{P}{M}=p=\frac{Q}{m}=q \text { ist. }
$$

B. Nun setze man, $\boldsymbol{M}$ und $\boldsymbol{Q}$ seien nicht da, sondern blofs $\boldsymbol{P}$ und $\boldsymbol{m}$; das heifst: $\boldsymbol{P}$ wirke allein, mittelbar durch den Hebel $\boldsymbol{A C B}$, auf die Masse $\boldsymbol{n}$ : so mufs, wenn $\boldsymbol{P}$ auf $m$ dieselbe Wirkung hervorbringen soll, wie wenn es olne den Hebel unmittelbar auf $\boldsymbol{M}$ wirkte, $m=M \cdot \frac{A C}{B C}$ oder, wenn man

$$
\text { (25.) } A C=a, \quad B C=b-\text { und } \frac{b}{a}=k
$$

setzt,

$$
\text { (26.) } m=\frac{M}{K}
$$

sein. Denn gesetzt, die Kraft $\boldsymbol{P}$ treibe, unmittelbar auf $\boldsymbol{M}$ wirkend, mit ihrer beschleunigenden Kraft $\boldsymbol{p}$, den Punct $\boldsymbol{A}$ in der Zeit $\boldsymbol{T}$ durch die Länge des Kreisbogens $A \alpha=r$, so mufs es, mittelbar durch den Hebel auf $m$ wirkend, den Punct $\boldsymbol{B}$ durch die Länge des Kreisbogens $\boldsymbol{B} \beta=\boldsymbol{R}$ zu treiben vermögen, wo $\alpha C \beta$ eine gerade Linie ist. Und da nun

$$
\text { (27.) } \frac{B \beta}{A \alpha} \text { oder } \frac{R}{r}=\frac{b}{a}=k
$$

ist (25.), so mufs, vermöge (14. \$. 5. E.), $m=M \cdot \frac{r}{R}$, also nach (27.) $m=\frac{M}{k}$ sein; wie (26.).

$C$. Nun wirke andrerseits auf diese Masse $m$ allein die Kraft $\boldsymbol{Q}$ unmittelbar und mit derselben beschleunigenden Kraft $p$, wie $\boldsymbol{P}$ auf $\boldsymbol{M}$, und $\boldsymbol{P}$ und $\boldsymbol{M}$ seien nicht da, so wird $\boldsymbol{Q}$ die Masse $\boldsymbol{m}$ in der Zeit $\boldsymbol{T}$ ebenfalls durch den Raum $r(=A \alpha)$ treiben, wie $\boldsymbol{P}$ die Masse $\boldsymbol{M}$. Man nehme jetzt die 
Masse $m$ weg und setze links eine Masse $\boldsymbol{N}$, auf welche $\boldsymbol{Q}$ dieselbe Wirkung mittelbar durch den Hebel hervorbringen soll, wie auf $m$, so mufs sie diese Masse in der Zeit $\boldsymbol{T}$ durch den Raum $r \cdot \frac{A C}{B C}=r \cdot \frac{a}{b}=\frac{r}{k}$ zu treiben vermögen. Also mufs zufolge (14. \$.5. E.)

$$
\text { (28.) } \quad m r=N \cdot \frac{r}{k} \quad \text { odér } \quad N=m k,
$$

mithin vermöge (26.)

$$
\text { (29.) } \boldsymbol{N}=\boldsymbol{M}
$$

sein.

$\boldsymbol{D}$. Demnach vermag die Kraft $\boldsymbol{Q}$, welche mit der beschleunigenden Kraft $\boldsymbol{p}$ auf $\boldsymbol{m}$ unmittelbar wirken soll, mittelbar durch den Hebel, die Masse $\boldsymbol{M}$ links, in der Zeit $\boldsymbol{T}$ durch den Raum $\frac{\boldsymbol{r}}{\boldsymbol{k}}$ zu treiben; und zwar mit derjenigen beschleunigenden Kraft $\varphi_{1}$, die sie auf $\boldsymbol{M}$ hervorbringt. Dieselbe verhält sich zu $p$ nach (13. \$.5. D.) wie die durchlaufenen Räume $r$ und $\frac{r}{k}$, so dal's

$$
\text { (30.) } \frac{p}{q_{1}}=\frac{r}{r: k}=k \text { also } q_{1}=\frac{p}{k} \text { ist. }
$$

E. Mit dem $k$ fachen $k \cdot \frac{p}{l_{k}}=p$ dieser beschleunigenden Kraft $q_{1}=\frac{p}{k}$ würde in derselben Zeit $T$ die Masse $M$ nach (13. \$. 5. D.) durch das kfache $\boldsymbol{k} \cdot \frac{\boldsymbol{r}}{\boldsymbol{k}}=\boldsymbol{r}$ des Raums $\frac{\boldsymbol{r}}{\boldsymbol{k}}$ getrieben werden, durch welchen $\boldsymbol{Q}$ nach $\boldsymbol{D}$, mittelbar durch den Hebel, die Masse $\boldsymbol{M}$ treibt. Da nun $\boldsymbol{P}$, unmittelbar mit ihrer beschleunigenden Kraft $p=\frac{P}{M}$ auf $\boldsymbol{M}$ wirkend, das Gleiche thut, so bringt $Q$, mit ihrer der $p$ gleichen beschleunigenden Kraft $\frac{Q}{m}$ (24.) auf $m$ wirkend, wenn sie statt dessen mittelbar durch den Hebel auf $\boldsymbol{M}$ wirkt, auf die Masse $\boldsymbol{M}$ dieselbe Wirkung hervor, wie $\boldsymbol{P}$ unmittelbar; aber in entgegengesetzter Richtung. Also halten sich die bewegenden Kräfte $P=M p$ und $\boldsymbol{Q}=m p$, die mit gleichen beschleunigenden Kräften $\boldsymbol{p}$ auf die Massen $M$ und $\boldsymbol{m}$ wirken, das Gleichgewicht, wenn nach (26.)

(31.) $\frac{M}{m}=k=\frac{b}{a}$ oder auch $\frac{M p}{m p}$ oder $\frac{P}{Q}=\frac{b}{a}=\frac{B C}{A C}$ ist.

F. Läfst man den Hebel, statt aus der geraden unbiegsamen Linie $\boldsymbol{A C B}$, aus zwei festen rechtwinkligen Dreiecken $C A A_{1}$ und $C B B_{1}$ (Fig. 8) bestehen, die beide und in $C$ ebenfalls nicht biegsam sind, so ist es offenbar 
15. Vom Parallelogramm- und dem Parallelepipedum der Kräfte.

gleichgültig, ob die Kräfte $\boldsymbol{P}$ und $\boldsymbol{Q}$ in $\boldsymbol{A}$ und $\boldsymbol{B}$, oder in $\boldsymbol{A}_{1}$ und $\boldsymbol{B}_{1}$ angebracht sind. Da nun vermöge der Ähnlichkeit der Dreiecke $C A A_{1}$ und $\boldsymbol{C B} B_{1}$,

$$
\text { (32.) } \frac{B C}{A C}=\frac{B_{1} C}{A_{1} C}
$$

ist, so sind die Kräfte $\boldsymbol{P}$ und $\boldsymbol{Q}$ auch an dem Hebel $\boldsymbol{A}_{1} \boldsymbol{C B _ { 1 }}$ im Gleichgewicht, wenn

$$
\text { (33.) } \quad \frac{P}{Q}=\frac{B_{1} C}{A_{1} C}
$$

jst. Und dies ist der Satz vom Hebel.

$$
\text { A } n \text { m e } r k \text { u } \mathrm{g} \text {. }
$$

8.

A. Dafs die Beweise (\$. 6. und 7.) der Sätze vom Parallelogramm der Kräfte und vom Hebel Begriffe und Sätze aus der Dynamik vorausnehmen, ist allerdings ungewöhnlich; aber daraus folgt nicht, dafs es unangemessen sei. In der That ist die Sonderung der Statik von der Dynamik eigentlich doch fast nur etwas Willkürliches. Die Statik beschäftigt sich, in der Lehre von den Wirkungen der Kräfte (der Mechanik), mit einem besondern einzelnen Falle: dem, in welchem sich die Kräfte aufheben oder Gleichgewicht Statt findet, und von diesem besondern einzelnen Falle geht man zu dem Allgemeinen, der Dynamik, weiter. Mag nun gleich das Verfahren, vom Besondern zum Allgemeinen aufzusteigen, allerdings für den Unterricht in der Regel das passendste sein, so ist doch auch der umgekehrte Weg keinesweges verboten; und es giebt der Fälle mehrere, wo man durch das Allgemeine für das Besondere leichter zum Ziele kommt, als unmittelbar. Zum Beispiel: aus dem allgeineinen Ausdruck einer veränderlichen Gröfse lassen sich ihre culminirenden Werthe (ihre Maxima und Minima) viel leichter finden, als unmittelbar. Stellte man in der Algebra den allgemeinen Taylorschen Satz (von der Entwicklung einer Function durch Differenzen) an die Spitze, so hätte man Manches, was ohne ihn viel Mühe macht, sehr leicht; wie z. B. den allgemeinen binomischen Lehrsatz, und Anderes; und zugleich wäre schon zu der Differentialrechnung der Grund gelegt. In der Planimetrie leisten bekanntlich stereometrische Sätze, z. B. bei der Untersuchung der Schneidepuncte von Linien, gute Dienste u. s. w. Hier oben zeigt es sich, dafs man durch dynamische Sätze zu einem vom Hebel unabhängigen Beweise des Satzes vom Parallelogramm der Kräfte offenbar einfacher und leichter gelangt, als vielleicht 
auf irgend eine Weise durch blofs statische Mittel. Es wäre vielleicht gar nicht unangemessen, in der Mechanik, und eben so in der Analysis und Geometrie, die ersten Grundzüge dieser drei Theile der Mathematik, in so weit es für das weiter erst abzuhandelnde Besondere nützlich sein kann, an die. Spitze zu stellen, darauf erst das Besondere, die Buchstabenrechnung und Algebra, die Planimetrie und die Statik weiter abzuhandeln, und dann zu dem Allgemeineren überzugehen.

$\boldsymbol{B}$. Sollte indessen das obige Verfahren nicht beliebt werden, besonders nicht für den Beweis des Satzes vom Hebel, und man bestände auf blofs statische Beweise, so ist für diesen Fall zu bemerken, dafs es jedenfalls besser sein wird, erst den Satz vom Hebel, unabhängig von dem vom Parallelogramm der Kräfte, zu beweisen, und dann aus ihm den Beweis des Satzes vom Parallelogramm der Kräfte herzuleiten; (was nach (\$. 2.) elementar und leicht geschieht): besser, als umgekehrt nach (\$. 3.) den Satz vom Hebel auf irgend einen unabhängigen statischen Beweis des Satzes vom Parallelogramm der Kräfte zu gründen. Denn von den unabhängigen Beweisen der beiden Sätze ist jedenfalls der vom Hebel der leichteste.

C. Gewöhnlich bedürfen die unabhängigen, blofs statischen Beweise beider Sätze, wie in ( $\$$. 1.) bemerkt, des Überganges vom Rationalen sum Irrationalen. Allein es dürfte bei dem Beweise des Satzes vom Hebel vielleicht nicht unmöglich sein, diesen Übergang zu ersparen. Nemlich auf folgende Weise.

Unabhängiger statischer Beweis des Satzes vom Hebel, ohne den Übergang vom Rationalen zum Irrationalen.

9.

A. Es sei $\boldsymbol{A C B}$ (Fig. 9) eine unbiegsame gerade Linie, aber nicht ohne Masse, sondern mit Masse, die gleichförmig vertheilt ist, so dafs gleiche Längentheile der Linie gleichviel Masse enthalten. Ferner sollen alle gleichen Theile der Masse von gleichen Kräften, alle in Richtungen, die auf $\boldsymbol{A C B}$ senkrecht sind, nach unten getrieben werden; jeder Theil der Masse $\boldsymbol{M}$ der Linie soll von derselben beschleunigenden Kraft $p$ getrieben werden, so dafs die gesammte bewegende Kraft $\boldsymbol{P}$, welche die Masse $\boldsymbol{M}$ der Linie nach unten treibt,

$$
\text { (34.) } \boldsymbol{P} \doteq \boldsymbol{p} M
$$

ist. Der Einwand etwa, dafs es eine unbiegsame blo/se Linie mit Masse Crelle's Journal f. d. M. Bd. XLIV. Heft 3. 
in der Wirklichkeit nicht gebe, ist nicht gültig; denn es giebt in der Wirklichkeit auch keine unbiegsame Linie ohne Masse, und überhaupt keine blofse Linie. Mit demselben Rechte aber, wie solche Linien vorausgesetzl werden, kann auch eine unbiegsame gerade Linie mit Masse vorausgeselzt werden.

$\boldsymbol{B}$. Da die Kraft $\boldsymbol{P}$ die Linie $\boldsymbol{A C B}$, senkrecht auf sie, nach unten zieht, so wird offenbar eine in $\operatorname{der}$ Mille $C$ von $\boldsymbol{A B}$ senkrecht auf $\boldsymbol{A C B}$ uach oben ziehende Kraft $C C_{1}=P$ allen den Kräften, die auf die Masse der Linie wirken, das Gleichgewicht halten, und die Linie wird, von $\boldsymbol{P}$.gleichsam getragen, vollkommen in Ruhe bleiben. Denn da alle Wirkungen auf die Masse der Linie auf beiden Seiten von $C$ vollkommen dieselben sind, so ist kein Grund vorhanden, weshalb sich die Linie um den durch die Kraft $\boldsymbol{P}$ gleichsam fest gewordenen Punct drehen sollte. Aus demselben Grunde, aus welchem $\boldsymbol{A}$ z. B. nach unten sich bewegen möchte, müfste sich auch $\boldsymbol{B}$ ebenfalls nach unten bewegen; und da beides zugleich wegen der Unbiegsamkeit der Linie nicht angeht, so bleibt die Linie in Ruhe.

C. Läfst man nun der Kraft $\boldsymbol{C} C_{1}=P$ eine gleiche Kraft $C C_{2}=P$ in gerade entgegengesetzter Richtung entgegenwirken, die also jener das Gleichgewicht häl, so folgt, dafs diese Kraft $\boldsymbol{C} C_{2}=\boldsymbol{P}$ die Linie eben so nach unten zieht, wie die auf die Masse der Linie gleichförmig vertheilte Kraft $\boldsymbol{P}=\boldsymbol{M} \boldsymbol{M}$ (34.). Die Wirkung ist dieselbe, und man kann daher, statt die Linie mit Masse und mit gleichförmig auf dieselbe wirkenden Kräften anzunehmen, auch setzen, die unbiegsame Linie sei ohne Masse und in ihrer Mitte treibe eine Kraft $\boldsymbol{P}$ sie nach unten.

D. Nun sei $\boldsymbol{A B}$ (Fig. 10) eine unbiegsame gerade Linie, wiederum mit gleichförmig vertheilter Masse, auf welche überall die beschleunigende Kraft $\boldsymbol{p}$ wirkt. Man theile die Länge $\boldsymbol{A B}$ der Linie in zwei willkürliche Theile $\boldsymbol{A D}=\boldsymbol{m}$ und $\boldsymbol{B D}=\boldsymbol{n}$, die sich also zu einander verhalten wie $\boldsymbol{m}$ zu $n$, so dafs

$$
\text { (35.) } \quad \frac{A D}{B D}=\frac{m}{n}
$$

ist; wo $m$ und $n$ eben so wohl irrational, als ganze und gebrochene Zahlen sein können.

E. Nimmt man jetzt die unbiegsame Linie ohne Masse an, und setzt nach (C.), statt der Käfte, mit welchen die Theile $\boldsymbol{A D}$ und $\boldsymbol{B D}$, wenn sie Masse haben, nach unten gezogen werden, in der Mitle der Linien und senkrecht auf $\boldsymbol{A B}$ zwei Kräfte $\boldsymbol{P}$ und $\boldsymbol{Q}$ angebracht, die zufolge (C.) die Theile $\boldsymbol{A D}$ und 
$\boldsymbol{B D}$ ganz eben so nach unten ziehen, und die also jenen Kräften gleich sind, so müssen sich diese beiden Kräfte $\boldsymbol{P}$ und $\boldsymbol{Q}$ wie die Längen $\boldsymbol{A D}$ und $\boldsymbol{B D}$ verhalten: denn so verhalten sich die Kräfte, welche die Theile mit Masse nach unten ziehen, weil auf jeden gleichen Theil der Länge die gleiche Kraft kommt: also mufs

$$
\text { (36.) } \quad \frac{P}{Q}=\frac{m}{n}=\frac{A D}{B D} \text { sein. }
$$

F. Aber auch der Kraft, welche die ganze Linie $\boldsymbol{A B}$, falls sie Masse hat, nach unten treibt, hält, zufolge $\boldsymbol{B}$ in ihrer Mitte $\boldsymbol{C}$ eine senkrecht auf $\boldsymbol{A B}$ nach oben ziehende Kraft $\boldsymbol{C} C_{1}$ das Gleichgewicht, wenn diese Kraft eben so stark ist, wie die Kraft, welche die ganze Linie, mit Masse, nach unten treibt. Und da nun der Theil $\boldsymbol{A D}$, mit Masse, von der Kraft $\boldsymbol{P}$ und der Theil $\boldsymbol{B D}$, mit Masse, von der Kraft $\boldsymbol{Q}$, also die ganze Linie, mit Masse, zusammen mit der Kraft $\boldsymbol{P}+\boldsymbol{Q}$ nach unten getrieben wird, so hält eine Kraft $\boldsymbol{C} \boldsymbol{C}_{1}=\boldsymbol{P}+\boldsymbol{Q}$, in der Mitte $\boldsymbol{C}$ von $\boldsymbol{A B}$, senkrecht auf $\boldsymbol{A B}$ nach oben ziehend, den Kräften, mit welchen die ganze Linie, mit Masse, nach unten getrieben wird, das Gleichgexicht, folglich auch den beiden Kräften $\boldsymbol{P}$ und $\boldsymbol{Q}$ in der Milte $\boldsymbol{F}^{\top}$ und $\boldsymbol{E}$ von $\boldsymbol{A D}$ und $\boldsymbol{B D}$, welche die ganze Linie ohne Masse eben so stark nach unten ziehen.

G. Nun ist $\boldsymbol{A D}=m, \boldsymbol{B D}=n, \boldsymbol{A B}=m+n$, also $\boldsymbol{A C}=\frac{1}{2}(m+n)$, $\boldsymbol{A H}=\boldsymbol{D H}=\frac{1}{2} \boldsymbol{m}$ und $\boldsymbol{B E}=\boldsymbol{E D}=\frac{1}{2} \boldsymbol{n}$ und folglich $\boldsymbol{F C}=\boldsymbol{A C}-\boldsymbol{A F}$ $=\frac{1}{2}(m+n)-\frac{1}{2} m=\frac{1}{2} n$ und $E C=B C-B E=\frac{1}{2}(m+n)-\frac{1}{2} n=\frac{1}{2} m$, also ist

$$
\text { (37.) } \frac{E C}{F C}=\frac{\frac{1}{2} m}{\frac{1}{2} n}=\frac{m}{n}
$$

und vermöge (36.)

$$
\text { (38.) } \quad \frac{E C}{F C}=\frac{P}{Q} \text {; }
$$

und dies ist der Salz vom senkrechten Hebel. Dafs der Ruhepunct $C$ von der Kraft $\boldsymbol{P}+\boldsymbol{Q}$ gedrückt werde, zeigt sich in $(\boldsymbol{F}$.$) . Der Übergang zum$ schiefen Hebel kann wie in (\$.7. F.) geschehen.

\section{0.}

Will man die Vorstellung einer unbiegsamen geraden Linie nicht mit Masse, sondern nur ohne Masse gestatten (wozu es freilich keinen hinreichenden Grund geben dürfte), so ist, auf die Weise wie z. B. Poinsot in seiner Statik verfährt, zuerst zu beweisen: 
a. Dafs zweien gleichen Kräften $\boldsymbol{P}$ und $\boldsymbol{P}$, die in gleichen Entfernungen $\boldsymbol{A C}=\boldsymbol{B C}$ von $\boldsymbol{C}$ (Fig. 9) in parallelen Richtungen auf einen Hebel 'wirken, von einer Kraft $2 \boldsymbol{P}$, die in $\boldsymbol{C}$, zuischen $\boldsymbol{A}$ und $\boldsymbol{B}$, parallel mit $\boldsymbol{P}$ und $\boldsymbol{P}$ diesen gerade entgegenwirkt, das Gleichgewicht gehalten wird. Dies geschieht durch Zusammensetzung und Wiederserlegung von Kräften, auf die Weise wie in (\$. 2. F. G. H.), also mit Hülfe und Vorausnahme von Bęriffen und Sätzen, welche schon zum Beweise des. Satzes vom Parallelogramm der Kräfle gehören.

b. Ist Dies bewiesen, so folgt weiter, dafs, wenn man $\boldsymbol{A B}$ (Fig. 10) z. B. in $m+n$ gleiche Theile theilt (wo $m$ und $n$ ganze Zahlen sind) und je mitten zwischen zwei Theilungspuncten eine gleiche Kraft $p$, desgleichen alle diese $\boldsymbol{m}+\boldsymbol{n}$ Kräfte in parallelen Richtungen auf $\boldsymbol{A B}$ wirken läfst: dafs dann eine in der Mitte $\boldsymbol{C}$ von $\boldsymbol{A B}$, parallel mit den Kräften $p$, in entgegengesetzter Richtung auf $\boldsymbol{A B}$ wirkende Kraft $(\boldsymbol{m}+\boldsymbol{n}) \boldsymbol{p}$ allen den $\boldsymbol{m}+\boldsymbol{n}$ Kräften das Gleichgewicht hält. Desgleichen, dafs, wenn von den $m+n$ gleichen Theilen von $\boldsymbol{A B} m$ Theile auf $\boldsymbol{A D}$ und $\boldsymbol{n}$ Theile auf $\boldsymbol{B D}$ kommen, eine Kraft $\boldsymbol{P}=\boldsymbol{m p}$ in der Mitte $\boldsymbol{F}$ von $\boldsymbol{A D}$ und eine Kraft $\boldsymbol{Q}=n \boldsymbol{p}$ in der Mitte $\boldsymbol{E}$ von $\boldsymbol{B D}$, parallel mit den $p$ wirkend, eben so viel thun, wie die $m$ Kräfte $p$, die auf $\boldsymbol{A D}$ und die $\boldsymbol{n}$ Kräfte $\boldsymbol{p}$, die auf $\boldsymbol{B D}$ wirken, so dafs also die Kraft $(\boldsymbol{m}+\boldsymbol{n}) \boldsymbol{p}$ in $\boldsymbol{C}$ den beiden in $\boldsymbol{F}$ und $\boldsymbol{E}$ wirkenden Kräften $\boldsymbol{P}$ und $\boldsymbol{Q}$ das Gleichgewicht hält.

c. Hierauf wird endlich, ganz ähnlich wie in (\$. 9. D. bis G.), bewiesen, dafs sich $\boldsymbol{P}$ und $\boldsymbol{Q}$ zu einander verhalten müssen, wie $\boldsymbol{E C}$ zu $\boldsymbol{F C}$, oder wie $m$ zu $n$.

d. Aber da vorausgesetzt worden, dafs $m$ und $n$ ganze Zahlen sind, so wird der Salz vom Hebel auf diese Weise nur erst für den Fall bewiesen, wo $\frac{m}{n}$ rational ist. Um zu den Fall, wo $\frac{m}{n}$ irrational ist, überzugehen, ist zunächst zu beweisen, dafs, wenn z. B. in (Fig. 10) $\boldsymbol{Q}$ zunimmt, während $\boldsymbol{P}$ bleibt, der Ruhepunct $\boldsymbol{C}$ des Hebels von $\boldsymbol{F}$ sich weiter entfernt, also dafs $\boldsymbol{F C}$ mit $\boldsymbol{Q}$ zugleich wächst. Dies folgt aus dem Umstande, dafs, wie sich bei dem Theile des Beweises (\$. 2. F. G. H.) ergeben hat, der Ruhepunct des Hebels immer zwischen den Angriffspuncten der beiden Kräfte $\boldsymbol{P}$ und $\boldsymbol{Q}$, die auf ihn wirken, liegen mufs: das heifst, dafs der Angriffspunct $C$ einer Kraft $\boldsymbol{R}=\boldsymbol{P}+\boldsymbol{Q}$, die nach $\boldsymbol{C} \boldsymbol{C}_{2}$ auf den Hebel $\boldsymbol{F} \boldsymbol{E}$ eben so wirkt wie $\boldsymbol{P}$ und $\boldsymbol{Q}$, immer zwischen $\boldsymbol{E}$ und $\boldsymbol{F}$ fällt; was auch $\boldsymbol{P}$ und $\boldsymbol{Q}$ sein mögen. 
Vermöge dessen wird, da $\boldsymbol{R}=\boldsymbol{P}+\boldsymbol{Q}$ in $\boldsymbol{C}$ so viel thut als $\boldsymbol{P}$ und $\boldsymbol{Q}$ in $\boldsymbol{F}$ und $\boldsymbol{E}$, falls $\boldsymbol{Q}$ z. B. um $\boldsymbol{K}$, zunimmt, eine Kraft $\boldsymbol{R}+\boldsymbol{K}=\boldsymbol{P}+\boldsymbol{Q}+\boldsymbol{K}$ so viel thun als $\boldsymbol{P}$ und $\boldsymbol{Q}+\boldsymbol{K}$. Aber $\boldsymbol{R}$ in $\boldsymbol{C}$ thut schon so viel als $\boldsymbol{P}+\boldsymbol{Q}$, also mufs die neue Kraft $\boldsymbol{R}+\boldsymbol{K}$ so viel thun als $\boldsymbol{R}$ und $\boldsymbol{K}$, und der Angriffspunct dieser Kraft $\boldsymbol{R}+\boldsymbol{K}$ liegt zwischen $\boldsymbol{C}$ und $\boldsymbol{E}$ : also rückt der Ruhepunct des Hebels $\boldsymbol{E}$ näher, wenn $\boldsymbol{Q}$ zunimmt, und $\boldsymbol{F} \boldsymbol{C}$ und $\boldsymbol{Q}$ nehmen zugleich zu.

$e$. Nachdem auch Dies bewiesen worden, schreitet man nun auf die gewöhnliche Weise zu dem Beweise des Überganges von der Rationalität zur Irrationalität von $\frac{m}{n}$.

Aber die Wiederholung dieses Beweises, auch hier in diesem Falle, ist völlig unnöthig, da der Beweis leicht allgemein und ein für allemal für alle ähnliche Fälle gegeben werden kann, die in der Rechenkunst und Geometrie, eben so wohl wie in der Mechanik, häufig vorkommen. Wieder/oolt man ihn in jedem ähnlichen Falle, so thut man in der That nicht viel anders, als wenn man z. B. den Beweis dieser oder jener Gleichung, der allgemein durch Buchstaben gegeben werden kann, stets für diese oder jene besondern Zahlenwerthe der Buchstaben wiederholt.

Ich habe hierauf schon in meinem Lehrbuche der Elemente der Geometrie (Berlin, bei G. Reimer. 1826.) aufmerksam gemacht und im ersten Bande dieses Buchs (S. 125) den allgemeinen Beweis gegeben. Für den Fall, dafs Dies nicht beachtet sein sollte, setze ich die Stelle, welche den Beweis enthält, hieher, nur mit veränderter Buchstabenbezeichnunğ und noch etwas vervollsı ändigt.

Übergang vom Rationalen zum Irrationalen.

\section{1.}

„Erklärung. Wenn ungleichartige Gröfsen, z. B. Linien und Winkel, „Winkel und Flächen, Winkel und Körper" (hier Linien und Kräfte), „die "auf irgend eine Weise von einander abhangen, die Eigenschaft haben, dafs, „so lange die eine wächst, oder abnimmt, die andere ebenfalls immer wächst, „oder immer abnimmt, und umgekehrt, nie die zweite vom Wachsen zum "Abnehmen übergeht, so lange es nicht die erste auch thut, so sollen die „Gröfsen zusammengehörig heifsen; und zwar, wenn sie beide zugleich ,immerfort wachsen, oder immerfort abnehmen, gleichförmig-zusammengehörig, „wenn die eine abnimmt, während die andere wächst, entgegengesetzt-zusan"mengehörig". 
„Lehrsatz. Wenn von zwei ungleichartigen, aber gleichförmig„zusammengehörigen, von einander abhängigen Gröfsen $c$ und $a$ bewiesen "werden kann, dafs während $c$ in das $k$ fache $c$ oder in $k c=c_{1}$ übergeht, "aus $a$ ebenfalls grade das $k$ fache $k a$ oder $k a=a_{1}$ wird, wo $k$ eine ganze "Zahl oder einen rationalen Bruch bedeutet, so dafs also $c_{1}=a_{1}$ commen„surable Gleichvielfache von $c$ und $a$ sind, so gilt das Nämliche auch, wenn ,kc mit $c$ incommensurabel, oder wenn $k$ eine irrationale Zahl ist; das „heifst: auch dann gehört grade $k a$ zu $k c$, und $k c$ und $k a$ sind also immer „Gleichvielfuche von $c$ und $a, k$ mag rational oder irrational sein, milhin „für jede beliebige Zahl $k$."

„Beweis. Ginge, im Fall $k$ irrational ist, a nicht grade in $k a$ über, "wenn $c$ in $k c$ übergeht, so ginge es in eine grö/sere oder kleinere Gröfse „als ka über, z. B. in die grö/sere $(k+e) a$; wo $e$ irgend eine" (rationale oder irrationale) „Zahl ist. Es lassen sich aber" (rationale) „Zahlen, nament„lich Brüche, so nahe bei einander annehmen, als man will; z. B. zwei Brüche ${ } p$ und $q$, die um weniger als $e$ von einander verschieden sind, was auch "e sein mag. Nun könnęn zwischen den beiden Zahlen $p$ und $q$ nicht zwei „andere zugleich liegen, deren Unterschied gröfser ist, als der Unterschied „von $p$ und $q$ : also lassen sich Brüche $p$ und $q$ annehmen, zwischen welchen ,$k$ und $k+e$ nicht zugleich liegen können.' Ist daher $p$. kleiner und $q$ gröfser „als $k$, so liegt $q$ nothwendig zwischen $k$ und $k+e$, und folglich ist qa grö/ser „als $k a$ und kleiner als $(k+e) a$; desgleichen ist qc grö/ser als $k c . "$

„Nun nehme man an, $c$ und $a$ wachsen, und $c$ gehe zuerst in $q c$ über, „so mufs nothwendig $q a$ aus $a$ werden, weil der Voraussetzung nach be„wiesen werden kann, dafs für alle ganzen Zahlen und Brüche, wie q, " $\boldsymbol{a}$ in das Gleichvielfache übergeht. Geht darauf weiter $c$ von $q c$ in $k c$ über, „so müfste $q a$ in $(k+e) a$ übergehen, weil nach der obigen Voraussetzung, „wenn $k c$ aus $c$ wird, $a$ in $(k+e) a$ übèrgeht. Aber $q c$ ist grö/ser als $k c$, "hingegen $q a$ ist kleiner als $(k+e) a$ : also würde die einè Gröfse $c$ von $q c$ „nach kc abnehmen, während die andere von qa bis $(k+e) a$ wächst. Dieses „ist der Voraussetzung entgegen, weil die Grölsen $c$ und a gleichförmig „zusammengehören, also immer nur zugleich wachsen oder abnehmen sollen, „nie abwechselnd. Folglich ist es unmöglich, dafs a in etwas Grö/seres „als ka übergeht, wäbrend $c$ von $c$ nach $k c$ gelangt."

"Auf ähnliche Art wird besiesen, dafs aus a nichts Kleineres als ka „werden kann, wenn $k c$ aus $c$ wird. Also mufs nothwendig immer a in 
${ }_{,} k a=a_{1}$ übergehen, wenn $k c=c_{1}$ aus $c$ wird; auch wenn $k$ irralional ist; „das heifst: auch dann sind $c_{1}$ und $a_{1}$ Gleichvielfache von $c$ und $a$. Folglich „gilt die Gleichvielfachheil, die für gleichförmig zusammengehörige commen"surable Gröfsen bewiesen wurde, auch ohne Ausnahme für gleichförmig "zusammengehörige incommensurable Gröfsen."

Für entgegengesetzt-zusammengehörige Gröfsen ist der Beweis ohne Weiteres in dem vorigen enthalten. Denn man darf nur, wenn $c$ und $a$ entgegengesetzt-zusammengehörige Gröfsen sind, z. B. $b-a$ stalt $a$ schreiben, wo $b>a$ und willkürlich ist. Alsdann sind $c$ und $b-a$ gleichförmig-zusammengehörige Gröfsen, und was von $b-a$ wie oben bewiesen wird, gilt auch von $a$.

Anwendung auf den Beweis des Satzes vom Hebel (\$. 10.).

12.

A. Hier ergab sich in (\$.10. d.), dafs FC (Fig. 10) immer wächst, also $E C$ immer abnimmt, folglich auch $\frac{F C}{E C}$ immer sunimmt, wenn $Q z u-$ nimmt, während $\boldsymbol{P}$ und $\boldsymbol{F} \boldsymbol{E}$ Dasselbe bleiben. Setzt man daher

$$
\text { (39.) } \frac{F C}{E C}=a \text { und } \frac{Q}{P}=c \text {, }
$$

so sind die von einander abhangenden ungleichartigen Gröfsen $a$ und $c$ gleichförmiy-zusammengehörige Gröfsen (\$.11.).

B. Nun ist ferner bewiesen worden; dafs

$$
\text { (40.) } \quad \frac{F C}{E C}=\frac{n}{m}=a
$$

für alle ganze Zahlen $m$ und $n$ gleich $\frac{Q}{P}=c$ ist: also dafs die rationalen Zahlen $a$ und $c$ einander gleich sind. Demnach sind auch $k a$ und $k c$ einander gleich, insofern $k$ eine rationale Zahl ist, damit $k a$ und $k c$ ebenfalls rational sein mögen.

C. Es nehmen aber $\frac{F C}{E C}=a$ und $\frac{Q}{P}=c$ nach (A.) immer zugleich $\mathrm{zu}$, niemals das eine $\mathrm{ab}$, wenn das andere zunimmt. Also findet Das was von $k a$ und $k c$ gilt, wenn $k$ oder $k a$ und $k c$ rational sind, nach dem allgemeinen Beweise in (\$. 11.) auch dann Statt, wenn ka und kc irrational sind; folglich ist auch dann $k a=k c$. Das heifst: auch dann, wenn das Verhältnifs $k c$ der Kräfte $\boldsymbol{P}$ und $\boldsymbol{Q}$ zu einander irrational ist, ist dieses Verhälınifs dem $k a$ 
der Hebels-Arme um den Ruhhepunct umgekehrt gleich; was dann der vollständige Satz vom Hebel ist.

Da dieser vollständige Beweis des Satzes vom Hebel viel weitläuftiger ist, als der in (\$.9.), so dürfte es für die Elemente der Statik, wenn man darin nur statische Beweise zulassen will, rathsam sein, den Satz vom Hebel wie in (\$. 9.) und dann weiter mit Hülfe dieses Satzes den Satz vom Parallelogramm der Kräfte wie in (\$. 2.) zu beweisen. Dann ist auch der Übergang vom Rationalen zum Irrationalen nirgends nothwendig.

Einiges vom Parallelepipedum der Kräfte.

13.

Da zwei Kräfte $\boldsymbol{a}$ und $\boldsymbol{b}$, die in der Ebene des Papiers nach Richtung und Gröfse durch die Linien $\boldsymbol{A B}$ und $\boldsymbol{A C}$ (Fig. 11) vorgestellt werden, eben so auf den Punct $\boldsymbol{A}$ wirken, wie eine Kraft welche die Diagonal $\boldsymbol{A d}$ des ebenen Parallelogramms $\boldsymbol{A B C D}$ nach Richtung und Gröfse ausdrückt, so wirkt, wenn eine dritte Kraft $\boldsymbol{A E}=\boldsymbol{c}$ hinzukommt, die nicht in der Ebene des Papiers, sondern $\ddot{u} b e r$ derselben, von $\boldsymbol{A}$ aus schräg nach oben gerichtet ist, und man zieht $\boldsymbol{D H}=\boldsymbol{A E}$ mit $\boldsymbol{A E}$ im Raume parallel, so dafs $A D E H$ ein ebenes Parallelogramm ist, die durch die Diagonal $\boldsymbol{A H}$ dieses Parallelogramms nach Richtung und Gröfse vorgestellte Kraft eben so stark auf $\boldsymbol{A}$, wie die beiden Kräfte $\boldsymbol{A D}$ und $\boldsymbol{A E}=\boldsymbol{c}$, und folglich eben so stark auf $\boldsymbol{A}$, als alle drei Kräfte $\boldsymbol{A B}=\boldsymbol{a}, \boldsymbol{A C}=\boldsymbol{b}$ und $\boldsymbol{A E}=\boldsymbol{c}$ zusammen.

Die Raum-Dingonal $\boldsymbol{A H}$ des Parallelepipedums, welches entsteht, wenn man durch $a$ und $b, b$ und $c$ und $c$ und $a$ drei Ebenen legt, von welchen drei andere durch die Endpuncte $\boldsymbol{B}, \boldsymbol{C}$ und $\boldsymbol{E}$ von $\boldsymbol{a}, \boldsymbol{b}$ und $\boldsymbol{c}$ mit jenen parallel gelegte Ebenen die Parallelogramme $\boldsymbol{B D H F}=A C G E, C D G H$ $=\boldsymbol{A B E F}$ und $\boldsymbol{E G H F}=\boldsymbol{A C D B}$ abschneiden, stellt also nach Richtung und Gröfse die mittlere Kraft vor, welche auf $\boldsymbol{A}$ eben so wirkt, wie die drei, durch die in $\boldsymbol{A}$ zusammenlaufenden $\boldsymbol{K}$ (anten des Parallelepipedums nach Richtung und Gröfse vorgestellten drei Kräfte $\boldsymbol{A B}=a, A C=b$ und $\boldsymbol{A E}=\boldsymbol{c}$.

Wir wollen hier die mannichfaltigen geometrischen Eigenschaften dieses Parallelepipedums der Kräfte etwas vollständiger, als es gewöhnlich geschieht, und dabei so einfach und kurz als möglich entwickeln, weil Dies für die Elemente vielleicht von Nutzen sein könnte. 
13. Vom Parallelogramm- und dem Parallelepipedum der Kräfte.

\section{4.}

Was bei dem Gegenstande vorkommt, soll, wie folgt, bezeichnet werden.

$$
\text { A. L i n i e n. }
$$

a. Durch $\boldsymbol{d}_{a}=\boldsymbol{A} \boldsymbol{G}=\boldsymbol{B H}, \boldsymbol{d}_{b}=\boldsymbol{A F}=\boldsymbol{C H}, \boldsymbol{d}_{c}=\boldsymbol{A D}=\boldsymbol{E H}$ die den Kanten $a, b$ und $c$ gegenüberliegenden Diagonalen der Seitenflächen. Die Summe ihrer Quadrate sei

$$
\text { (41.) } \Sigma d^{2}=d_{a}^{2}+d_{b}^{2}+d_{c}^{2} \text {. }
$$

b. Durch $\delta_{\alpha}=\boldsymbol{C E}=\boldsymbol{D F}, \delta_{\beta}=\boldsymbol{E B}=\boldsymbol{G D}, \delta_{\gamma}=\boldsymbol{C B}=\boldsymbol{G F}$ die den Winkeln $\boldsymbol{C A E}, \boldsymbol{E A B}, \boldsymbol{C A B}$, in diesen Seitenflächen selbst, gegenüber liegenden Diagonalen. Die Summe ihrer Quadrate sei

$$
\text { (42.) } \Sigma \delta^{2}=\delta_{\alpha}^{2}+\delta_{\beta}^{2}+\delta_{\gamma}^{2} \text {. }
$$

c. Durch $\Delta_{1}=\boldsymbol{A H}, \Delta_{2}=\boldsymbol{B G}, \Delta_{3}=\boldsymbol{C F}, \Delta_{4}=\boldsymbol{D E}$ die vier RaumDiagonalen des Parallelepipedurns. Die Summe ihrer Quadrate sei

(43.) $\Sigma \Delta=\Delta_{1}^{2}+\Delta_{2}^{2}+\Delta_{3}^{2}+\Delta_{4}^{2}$.

d. Durch

$$
\text { (44.) } \Sigma k^{2}=a^{2}+b^{2}+c^{2}
$$

die Summe der Quadrate der drei verschiedenen Kanten.

B. W i n k e l.

e. Durch $\alpha=\boldsymbol{C A E}, \beta=\boldsymbol{E} \boldsymbol{A B}, \gamma=\boldsymbol{B} \boldsymbol{A C}$ die Winkel, welche die Kanlen $a, b, c$ an $\operatorname{der}$ Ecke $A$ einschliefsen. Die Winkel, welche dieselben drei Kanten an den andern Ecken einschliefsen, sind theils $\alpha, \beta, \gamma$ selbst, theils die Supplemente davon; wovon weiter unten.

$f$. Durch $\boldsymbol{A}, \boldsymbol{B}, \boldsymbol{C}$ die Winkel, welche die Seitenflächen an den Kanten $a, b, c$ einschliefsen.

g. Alle übrigen Winkel zwischen zwei zusammenstofsenden Linien sollen durch diese beiden Linien bezeichnet und es soll, wo es nöthig, noch zwischen dieselben die Linie gesetzt werden, welche dem Winkel gegenüber liegt, Alles zusammen in Klammern geschlossen; so dafs also z. B. CDB $=\left(\boldsymbol{a}, \delta_{\gamma}, \boldsymbol{b}\right), \boldsymbol{E} \boldsymbol{A D}=\left(\boldsymbol{c}, \Delta_{4}, \delta_{\gamma}\right), \boldsymbol{A M B}=\left(\Delta_{1}, a, \mathcal{A}_{2}\right)$ ist. U. s. w.

$$
\text { C. Flächen - Inhal te. }
$$

h. Durch $s_{1}=A B C D=E F G H, s_{2}=A E C G=B F D H, s_{3}=A B E F$ $=\boldsymbol{C G H D}$ die Inhalte der drei verschiedenen Seitenflüchen des Parallelépipedums, welche $a$ und $b, b$ und $c, c$ und $a$ zu Seiten haben; die Summen 
ihrer Quadrate durch

$$
\text { (45.) } \Sigma s^{2}=s_{1}^{2}+s_{2}^{2}+s_{3}^{2} \text {. }
$$

i. Durch $\boldsymbol{D}_{a, d_{a}}=\boldsymbol{A B G H}, \boldsymbol{D}_{b, d_{b}}=A \boldsymbol{C H F}, \quad \boldsymbol{D}_{c, d_{c}}=\boldsymbol{A E H D}$ und

$$
\mathfrak{D}_{a, \delta_{\alpha}}=\boldsymbol{C E F D}, \quad \mathfrak{D}_{b, \delta_{\beta}}=\boldsymbol{B E G D}, \mathfrak{D}_{c, \delta_{\gamma}}=\boldsymbol{C G F B} .
$$

Die Inhalte der sechs Diagonalflächen des Parallelepipedums mit $a, d_{a}, b, d_{b}$, $c, d_{c}$ und $a, \delta_{\alpha}, b, \delta_{\beta}, c, \delta_{\gamma}$ zu Seiten; die Summen ihrer Quadrate durch

(46.) $\quad \begin{cases}\text { 1. } & \Sigma \boldsymbol{D}^{2}=\boldsymbol{D}_{a, d_{a}}^{2}+\boldsymbol{D}_{b, d_{b}}^{2}+\boldsymbol{D}_{c, d_{c}}^{2} \text { und } \\ \text { 2. } & \Sigma \mathfrak{D}^{2}=\mathfrak{D}_{a, \delta_{\boldsymbol{\alpha}}}^{2}+\mathfrak{D}_{b, \delta_{\beta}}^{2}+\mathfrak{D}_{c, \delta_{\gamma}}^{2}\end{cases}$

k. Durch $\varphi_{1}=\boldsymbol{B C E}, \varphi_{2}=\boldsymbol{A D F}, \varphi_{3}=\boldsymbol{G D} \boldsymbol{A}$ und $\varphi_{4}=\boldsymbol{C H B}$ die Inhalte der vier durch die Endpuncte der Kanten $a, b, c$, den vier Ecken $\boldsymbol{A}, \boldsymbol{B}, \boldsymbol{C}, \boldsymbol{D}$ gegenüber, gelegten dreieckigen Flächen; die Summe ihrer Quadrate durch

$$
\text { (47.) } \Sigma \varphi^{2}=\varphi_{1}^{2}+\varphi_{2}^{2}+\varphi_{2}^{3}+\varphi_{4}^{2} \text {. }
$$

l. Durch $\psi_{1}=\boldsymbol{G D H}, \psi_{2}=\boldsymbol{C E H}, \psi_{3}=\boldsymbol{E H B}, \psi_{4}=\boldsymbol{A G H}$ die Inhalte der vier, den Ecken $\boldsymbol{A}, \boldsymbol{B}, \boldsymbol{C}, \boldsymbol{D}$ gegenüber, durch die Endpuncte der in denselben zusammenstofsenden Seiten-Diagonalen gelegten dreieckigen Flächen; die Summe ihrer Quadrate durch

$$
\text { (48.) } \Sigma \psi^{2}=\psi_{1}^{2}+\psi_{2}^{2}+\psi_{3}^{2}+\psi_{4}^{2} \text {. }
$$

Auch die Flächen $(h, i, k, l)$ selbst sollen durch $s, D, \mathfrak{D}, \varphi$ und $\psi$ bezeichnet werden.

$$
\text { D. R a u m - In halt e. }
$$

m. Durch $\boldsymbol{P}$ den Raum-Inhalt des Parallelepipedums.

n. Durch $p_{1}=A B C E, p_{2}=B A D F, p_{3}=C G D A, \quad p_{4}=D C H B$ die Raum-Inhalte der vier Pyramiden, welche $\varphi_{1}, \varphi_{2}, \varphi_{3}$ und $\varphi_{4}$ zu Grundflächen und $\boldsymbol{A}, \boldsymbol{B}, \boldsymbol{C}, \boldsymbol{D}$ zu Spitzen haben; die Summe ihrer Quadrate durch

$$
\text { (49.) } \Sigma p=p_{1}+p_{2}+p_{3}+p_{4} \text {. }
$$

o. Durch $q_{1}=A G D F, q_{2}=B C E H, q_{3}=C E H B, q_{4}=D A G H^{\prime}$ die Raum-Inhalte der vier Pyramiden, welche $\psi_{1}, \psi_{2}, \psi_{3}$ und $\psi_{4}$ zu Grundflächen und $\boldsymbol{A}, \boldsymbol{B}, \boldsymbol{C}, \boldsymbol{D}$ zu Spitzen haben; die Summe ihrer Quadrate durch

$$
\text { (50.) } \Sigma q=q_{1}+q_{2}+q_{3}+q_{4} \text {. }
$$

p. Der Kürze wegen soll die Gröfse

$$
\text { (51.) } 1-\cos \alpha^{2}-\cos \beta^{2}-\cos \gamma^{2}+2 \cos \alpha \cos \beta \cos \gamma
$$

$$
=4 \sin \frac{1}{2}(\alpha+\beta+\gamma) \sin \frac{1}{2}(\alpha+\beta-\gamma) \sin \frac{1}{2}(\alpha-\beta+\gamma) \sin \frac{1}{2}(-\alpha+\beta+\gamma)=\lambda^{2}
$$

gesetzt werden. Dafs die beiden Ausdrücke von $\lambda$ einander gleich sind, ist bekannt.

Durch diese Bezeichnungen ausgedrückt, gedenken wir folgender Sätze und ihrer Beweise. 
15.

Zunächst ist für die bequeme Aufstellung der Formeln Folgendes zu bemerken.

A. In jeder der vier verschiedenen Ecken $\boldsymbol{A}, \boldsymbol{B}, \boldsymbol{C}, \boldsymbol{D}$ stöfst eine Raumdiagonal mit den drei Kanten, mit drei Seitendiagonalen, mit drei Seitenwinkeln und mit drei Flächenwinkeln zusammen; die Kanten und die Seitenflächen unter verschiedenen Winkeln. Und zwar gehören zusammen:
1. $\Delta_{1}$ mit $a, b, c, \quad \alpha, \quad \beta, \quad \gamma, d_{a}, d_{b}, d_{c}, \quad A, \quad B, \quad C$;
2. $A_{2} \operatorname{mit} a, b, c, \quad \alpha, 2 \varrho-\beta, 2 \varrho-\gamma, d_{\alpha}, \delta_{\beta}, \delta_{\gamma}, \quad A, 2 \varrho-B, 2 \varrho-C$;
3. $\Delta_{3} \operatorname{mit} a, b, c, 2 \varrho-\alpha, \quad \beta, 2 \varrho-\gamma, \delta_{\alpha}, d_{b}, \delta_{\gamma}, 2 \varrho-A, \quad B, 2 \varrho-C$;
4. $\Delta_{4}$ mit $a, b, c, 2 \varrho-\alpha, 2 \varrho-\beta, \quad \gamma, \delta_{\alpha}, \delta_{\beta}, d_{c}, 2 \varrho-A, 2 \varrho-B, \quad C$.

Um daher Das, was für die erste Ecke Statt findet, auf die folgenden zu übertragen, darf man nur die Werthe der Buchstaben nach (52.) verändern.

B. Für Das, was für dieselbe Ecke Statt findet, darf man nur die Buchstaben in ihrer Ordnung weilerrrucken.

Es ist (\$. 14. a. und b.):

I.

$\left\{\begin{array}{l}\text { 1. } d_{a}^{2}=b^{2}+c^{2}+2 b c \cos \alpha, \\ \text { 2. } d_{b}^{2}=c^{2}+a^{2}+2 c a \cos \beta, \\ \text { 3. } d_{c}^{2}=a^{2}+b^{2}+2 a b \cos \gamma .\end{array}\right.$

$\begin{cases}\text { 1. } & \delta_{\alpha}^{2}=b^{2}+c^{2}-2 b c \cos \alpha, \\ \text { 2. } & \delta_{\beta}^{2}=c^{2}+a^{2}-2 c a \cos \beta, \\ \text { 3. } & \delta_{\gamma}^{2}=a^{2}+b^{2}-2 a b \cos \gamma .\end{cases}$

(55.) $\begin{cases}\text { 1. } & d_{a}^{2}+\delta_{\alpha}^{2}=2\left(b^{2}+c^{2}\right), \\ 2 . & d_{b}^{2}+\delta_{\beta}^{2}=2\left(c^{2}+a^{2}\right), \\ 3 . & d_{c}^{2}+\delta_{\gamma}^{2}=2\left(a^{2}+b^{2}\right) .\end{cases}$

(56.) $\Sigma d^{2}+\Sigma \delta^{2}+4 \Sigma k^{2}$.

a. Wenn nemlich $C C_{1}, E E_{1}, D D_{1}, F F_{1}$ und $H H_{1}$ auf $A B H$ senkrecht sind, so ist $B D_{1}=\cos \gamma$ und $D D_{1}=\sin \gamma$, also

(57.) $\quad A D^{2}=(a+b \cos \gamma)^{2}+b^{2} \sin \gamma^{2}=a^{2}+b^{2}+2 a b \cos \gamma=d_{c}^{2}$.

Dies ist der Ausdruck (53. 3.). Die andern (53. 1. und 2.) finden sich nach (\$. 15. B.).

b. So wie in (53.1.) $d_{a}$ mit $b, c$ und $\alpha$ zusammengehört, so gehört nach (52.3.) $\delta_{\alpha}$ mit $b, c$ und $2 \varrho-\alpha$ zusammen. Also erhält man (54.1.) aus (53. 1.), wenn man $2 \varrho-\alpha$ statt $\alpha$ setzt. Die andern Ausdrücke (54. 2. u. 3.) ergeben sich nach (\$.15. B.).

c. Die Ausdrücke (55. und 56.) folgen aus denen (53. und 54.) unmittelbar. 
248 15. Vom Parallelogramm- und dem Parallelepipedum der Kr ̈̈fte.

Es ist für (\$.14. c.):

\section{II.}
(58.)
$\begin{cases}\text { 1. } & \Delta_{1}^{2}=\Sigma k^{2}+2 a b \cos \gamma+2 b c \cos \alpha+2 c a \cos \beta, \\ \text { 2. } & \Delta_{2}^{2}=\Sigma k^{2}-2 a b \cos \gamma+2 b c \cos \alpha-2 c a \cos \beta, \\ \text { 3. } \Delta_{3}^{2}=\Sigma k^{2}-2 a b \cos \gamma-2 b c \cos \alpha+2 c a \cos \beta, \\ \text { 4. } \Delta_{4}^{2}=\Sigma k^{2}+2 a b \cos \gamma-2 b c \cos \alpha-2 c a \cos \beta .\end{cases}$
(59.) $\Sigma \Delta^{2}=4 \Sigma k^{2}$.



a. Denn in dem Dreiecke $\boldsymbol{A B H}$ ist

$$
A H^{2}=A B^{2}+B H^{2}-2 A B \cdot B H \cos A B H,
$$

das heifst, weil $\boldsymbol{A B H}=2 \varrho-\boldsymbol{H B H}_{1}$ ist :

$$
\text { (63.) } \quad \Delta_{1}^{2}=a^{2}+d_{a}^{2}+2 a \cdot d_{a} \cos H B H_{1} \text {. }
$$

Nun ist $\boldsymbol{B} D_{1}=A C_{1}=b \cos \gamma$, und da $\boldsymbol{D H}=\boldsymbol{c}$ mit $\boldsymbol{A E}=\boldsymbol{c}$ im Raume parallel ist, $D_{1} H_{1}=A E_{1}=c \cos \beta$; also ist $B D_{1}+D_{1} H_{1}$ oder $B H_{1}=$ $b \cos \gamma+c \cos \beta$ und folglich, da $B \dot{H}_{1}=H B \cos H B H_{1}=d_{a} \cos H B H_{1}$ ist,

(64.) $\quad d_{a} \cos H B H_{1}=b \cos \gamma+c \cos \beta$.

Dies, nebst $d_{a}^{2}=b^{2}+c^{2}+2 b c \cos \alpha(53,1$.$) , in (63.) gesetzt, giebt$

(65.) $\quad \Delta_{1}^{2}=a^{2}+b^{2}+c^{2}+2 b c \cos \alpha+2 a b \cos \gamma+2 c a \cos \beta$;

wie (58.). Hierin weiter nach (52. 2.3.4.) $\alpha, 2 \varrho-\beta, 2 \rho-\gamma ; 2 \varrho-\alpha, \beta, 2 \varrho-\gamma$ und $2 \varrho-\alpha, 2 \varrho-\beta$ und $\gamma$ statt $\alpha, \beta, \gamma$ gesetzt, giebt (58. 2.3.4.).

b. Der Ausdruck (59.) folgt unmittelbar aus (58.), und (60.61.u. 62.) folgen aus (53. 54. u. 56.).

Es ist für (\$. 14. h.):

III.

(66.) $\quad s_{1}=a b \sin \gamma, \quad s_{2}=b c \sin \alpha, \quad s_{3}=c a \sin \beta$.

Z. B. $s_{1}$ ist gleich $A B \cdot C C_{1}=a b \sin \gamma$. Nach (\$. 15. B.) folgen die andern.

Es ist für (\$. 14. f.):

IV.

(67.) $\left\{\begin{array}{l}\text { 1. } \cos A=\frac{\cos \alpha-\cos \beta \cos \gamma}{\sin \beta \sin \gamma}, \\ \text { 2. } \cos B=\frac{\cos \beta-\cos \gamma \cos \alpha}{\sin \gamma \sin \alpha} \\ \text { 3. } \cos C=\frac{\cos \gamma-\cos \alpha \cos \beta}{\sin \alpha \sin \beta}\end{array}\right.$

$\left\{\begin{array}{l}\text { 1. } \sin A=\frac{\lambda}{\sin \beta \sin \gamma}, \\ \text { 2. } \sin B=\frac{\lambda}{\sin \gamma \sin \alpha}, \\ \text { 3. } \sin C=\frac{\lambda}{\sin \alpha \sin \beta} \text {. }\end{array}\right.$ 
a. Zieht man $\boldsymbol{Y X}$ und $\boldsymbol{Z} \boldsymbol{X}$, wo $\boldsymbol{A X}=1$ gesetzt wird, auf $\boldsymbol{A E}=\boldsymbol{c}$ senkrecht, so ist der Winkel $\boldsymbol{Y} X \boldsymbol{Z}=\boldsymbol{C}$. Also ist in den Dreiecken $\boldsymbol{Y X Z}$ und $\boldsymbol{Y A Z}$ :

(69.) $\quad \boldsymbol{Y} \boldsymbol{Z}^{2}=\boldsymbol{Y} \boldsymbol{X}^{2}+\boldsymbol{Z} \boldsymbol{X}^{2}-2 \boldsymbol{Y} \boldsymbol{X} \cdot \boldsymbol{Z} \boldsymbol{X} \cos \boldsymbol{C}=\boldsymbol{Y} \boldsymbol{A}^{2}+\boldsymbol{Z} \boldsymbol{A}^{2}-2 \boldsymbol{Y} \boldsymbol{A} \cdot \boldsymbol{Z} \boldsymbol{A} \cos \gamma$ Aber es ist

(70.) $\quad \boldsymbol{Y} \boldsymbol{X}=\operatorname{tang} \alpha, \quad \boldsymbol{Z} \boldsymbol{X}=\operatorname{tang} \beta, \quad \boldsymbol{Y} \boldsymbol{A}=\sec \alpha, \quad \boldsymbol{Z} \boldsymbol{A}=\sec \beta$, also giebt (69.) $\operatorname{tang} \alpha^{2}+\operatorname{tang} \beta^{2}-2 \operatorname{tang} \alpha \operatorname{tang} \beta \cos C=\sec \alpha^{2}+\sec \beta^{2}$ $-2 \sec \alpha \sec \beta \cos \gamma$ oder $-2 \operatorname{tang} \alpha \operatorname{tang} \beta \cos C=2-2 \sec \alpha \sec \beta \cos \gamma$ oder mit $\cos \alpha \cos \beta$ multiplicirt,

(71.) $\cos C \sin \alpha \sin \beta=\cos \gamma-\cos \alpha \cos \beta$;

wie (67. 3.). Nach (\$. 15. B.) finden sich (67. 1. u. 2 ).

b. Aus (67. 1.) folgt

$\sin A^{2}=1-\left(\frac{\cos \alpha-\cos \beta \cos \gamma}{\sin \beta \sin \gamma}\right)=\frac{\sin \beta^{2} \sin \gamma^{2}-\cos \alpha^{2}+2 \cos \alpha \cos \beta \cos \gamma-\cos \beta^{2} \cos \gamma^{2}}{\sin \beta^{2} \sin \gamma^{2}}$

$=\frac{\left(1-\cos \beta^{2}\right)\left(1-\cos \gamma^{2}\right)-\cos \alpha^{2}+2 \cos \alpha \cos \beta \cos \gamma-\cos \beta^{2} \cos \gamma^{2}}{\sin \beta \sin \gamma^{2}}$

$=\frac{1-\cos \alpha^{2}-\cos \beta^{2}-\cos \gamma^{2}+2 \cos \alpha \cos \beta \cos \gamma}{\sin \beta^{2} \sin \gamma^{2}} ;$ also

(72.) $\sin A=\frac{\lambda}{\sin \beta \sin \gamma}$;

wie (68. 1.). Nach (\$. 15. B.) finden sich (68. 2. u. 3.).

Es ist für $(\$ \cdot 14 . g$.$) :$

V.

\section{1. $d_{a} \cos (d a, c)=c+b$}

(73.)

$\left\{\begin{array}{l}\text { 1. } d_{a} \cos \left(d_{a}, c\right)=c+b \cos \alpha, \\ \text { 2. } d_{b} \cos \left(d_{b}, a\right)=a+c \cos \beta,\end{array}\right.$

3. $d_{c} \cos \left(d_{c}, b\right)=b+a \cos \gamma$.

(75.)

1. $\delta_{\alpha} \cos \left(\delta_{\alpha}, c\right)=c-b \cos \alpha$,

2. $\delta_{\beta} \cos \left(\delta_{\beta}, a\right)=a-c \cos \beta$,

3. $\delta_{\gamma} \cos \left(\delta_{\gamma}, b\right)=b-a \cos \gamma$.

(77.)

1. $d_{a} \cos \left(d_{a}, b\right)=b+c \cos \alpha$,

2. $d_{b} \cos \left(d_{b}, c\right)=c+a \cos \beta$,

3. $d_{c} \cos \left(d_{c}, a\right)=a+b \cos \gamma$.

1. $\delta_{\alpha} \cos \left(\delta_{\alpha}, b\right)=b-c \cos \alpha$,

(79.)
1. $d_{\alpha} \sin \left(d_{a}, c\right)=b \sin \alpha$,

2. $d_{b} \sin \left(d_{b}, a\right)=c \sin \beta$,

3. $d_{c} \sin \left(d_{c}, b\right)=a \sin \gamma$.

1. $\delta_{\alpha} \sin \left(\delta_{\alpha}, c\right)=b \sin \alpha$,

2. $\delta_{\beta} \sin \left(\delta_{\beta}, a\right)=c \sin \beta$,

3. $\delta_{\gamma} \sin \left(\delta_{\gamma}, b\right)=a \sin \gamma$.

1. $d_{a} \sin \left(d_{a}, b\right)=c \sin \alpha$,

2. $d_{b} \sin \left(d_{b}, c\right)=a \sin \beta$,

3. $d_{c} \sin \left(d_{c}, a\right)=b \sin \gamma$.

1. $\delta_{\alpha} \sin \left(\delta_{\alpha}, b\right)=c \sin \alpha$,

2. $\delta_{\beta} \sin \left(\delta_{\beta}, c\right)=a \sin \beta$,

3. $\delta_{\gamma} \sin \left(\delta_{\gamma}, a\right)=b \sin \gamma$. 
15. Vom Parallelogramm- und dem Parallelepipedın der Kräfte.

(81.)

$$
\left(\begin{array}{c}
\text { 1. } \quad c d_{a} \cos \left(d_{a}, c\right)+a d_{b} \cos \left(d_{b}, a\right)+b d_{c} \cos \left(d_{c}, b\right)=\frac{1}{2}\left(\Lambda_{1}^{2}+\Sigma k^{2}\right) \\
=b d_{a} \cos \left(d_{a}, b\right)+c d_{b} \cos \left(d_{b}, c\right)+a d_{c} \cos \left(d_{c}, a\right),
\end{array}\right.
$$

2. $c d_{a} \cos \left(d_{a}, c\right)+a d_{\beta} \cos \left(\delta_{\beta}, a\right)+b \delta_{\gamma} \cos \left(\delta_{\gamma}, b\right)=\frac{1}{2}\left(d_{2}^{2}+\Sigma k^{2}\right)$

$$
=b d_{a} \cos \left(d_{a}, b\right)+c \delta_{\beta} \cos \left(\delta_{\beta}, c\right)+a \delta_{\gamma} \cos \left(\delta_{\gamma}, a\right) \text {, }
$$

3. $\quad c \delta_{\alpha} \cos \left(\delta_{\alpha}, c\right)+a d_{b} \cos \left(d_{b}, a\right)+b \delta_{\gamma} \cos \left(\delta_{\gamma}, b\right)=\frac{1}{2}\left(A_{3}^{2}+\Sigma k^{2}\right)$

$$
=b \delta_{\alpha} \cos \left(\delta_{\alpha}, b\right)+c d_{b} \cos \left(d_{b}, c\right)+a \delta_{\gamma} \cos \left(\delta_{\gamma}, a\right) \text {, }
$$

4. $\quad c \delta_{\alpha} \cos \left(\delta_{\alpha}, c\right)+a \delta_{\beta} \cos \left(\delta_{\beta}, a\right)+b d_{c} \cos \left(d_{c}, b\right)=\frac{1}{2}\left(\Delta_{4}^{2}+\Sigma k^{2}\right)$

$$
=b \delta_{\alpha} \cos \left(\delta_{\alpha}, b\right)+c \delta_{\gamma} \cos \left(\delta_{\gamma}, c\right)+a d_{c} \cos \left(d_{c}, a\right) .
$$

1. $c d_{a} \sin \left(d_{a}, c\right)+a d_{b} \sin \left(d_{b}, a\right)+b d_{c} \sin \left(d_{c}, b\right)=\Sigma s$,

2. $\quad c \delta_{\alpha} \sin \left(\delta_{\alpha}, c\right)+a \delta_{\beta} \sin \left(\delta_{\beta}, a\right)+b \delta_{\gamma} \sin \left(\delta_{\gamma}, b\right)=\Sigma s$,

(82.)

3. $b d_{a} \sin \left(d_{a}, b\right)+c d_{b} \sin \left(d_{b}, c\right)+a d_{c} \sin \left(d_{c}, a\right)=\Sigma s$,

4. $b \delta_{\alpha} \sin \left(\delta_{\alpha}, b\right)+c \delta_{\beta} \sin \left(\delta_{\beta}, c\right)+a \delta_{\gamma} \sin \left(\delta_{\gamma}, b\right)=\Sigma s$.

a. Es ist $A F_{1}=a+c \cos \beta=A F \cos \left(d_{b}, a\right)=d_{b} \cos \left(d_{b}, a\right)$, wie (73. 2.), und (73. 1. u. 73. 3.), aus (\$.15. B.).

b. Es ist $A F \sin F \boldsymbol{A B}=F F_{1}$, das heifst $d_{b} \sin \left(d_{b}, a\right)=c \sin \beta$, wie (74. 2.), und (74. 1. u. 74. 3.), aus (\$. 15. B.).

c. (75.) folgen aus (73. u. 52.), weil $\delta_{\alpha}$ und $c$ mit $2 \varrho-\alpha$ und $c, \delta_{\beta}$ und $b$ mit $2 \varrho-\beta$ und $b$, und $\delta_{\gamma}$ und $b$ mit $2 \varrho-\gamma$ und $b$ zusammengehören.

d. Auf dieselbe Weise folgt (76.) aus (74.).

e. Es ist $A D \cos D A B=A D_{1}=a+b \cos \gamma=d_{c} \cos \left(d_{a}, b\right)$, wie (77. 3.), und (77. 1. u. 2.) folgen nach (\$.15.,B.).

f. Es ist $\boldsymbol{A D} \sin \boldsymbol{D A B}=d_{c} \sin \left(d_{c}, a\right)=D D_{1}=b \sin \gamma$; wie (78. 3.), und (78. 1. u. 2.) folgen nach (\$. 15. B.).

g. (79. u. 80.) folgen wieder aus (77. und 78.), nach (52.).

h. Die Ausdrücke (81.) folgen unmittelbar aus (73, 75, 77, 79 und 58.).

i. Die Ausdrücke (82.) folgen aus (74, 76, 78, 80 und 66.).

\section{VI.}

Es ist für (\$.14. g.):

$\begin{aligned} & \text { (83.) } \begin{cases}2 . & d_{b} \cos \left(d_{b}, A_{1}, b\right)=-a \cos \gamma-c \cos \alpha, \\ \text { 3. } & d_{c} \cos \left(d_{c}, A_{1}, c\right)=-b \cos \alpha-a \cos \beta .\end{cases} \\ & \text { (84.) } \begin{cases}1 . & d_{a} \cos \left(d_{a}, \Delta_{2}, a\right)=+c \cos \beta+b \cos \gamma, \\ 2 . & \delta_{\beta} \cos \left(\delta_{\beta}, \Delta_{2}, b\right)=+a \cos \gamma-c \cos \alpha, \\ 3 . & \delta_{\gamma} \cos \left(\delta_{\gamma}, \Delta_{2}, c\right)=-b \cos \alpha+a \cos \beta .\end{cases} \end{aligned}$ 
3. $\delta_{\gamma} \cos \left(\delta_{\gamma}, \Delta_{3}, c\right)=-a \cos \beta+b \cos \alpha$.

(86.) $\begin{cases}\text { 1. } & \delta_{\alpha} \cos \left(\delta_{\alpha}, \Delta_{4}, a\right)=+c \cos \beta-b \cos \gamma, \\ 2 . & \delta_{\beta} \cos \left(\delta_{\beta}, \Delta_{4}, b\right)=-a \cos \gamma+c \cos \alpha, \\ \text { 3. } & \delta_{c} \cos \left(d_{c}, \Delta_{4}, c\right)=+b \cos \alpha+a \cos \beta .\end{cases}$

(87.)

1. $a d_{a} \cos \left(d_{a}, \Delta_{1}, a\right)+b d_{b} \cos \left(d_{b}, \Delta_{1}, b\right)+c d_{c} \cos \left(d_{c}, \Delta_{1}, c\right)=\Sigma k^{2}-\Delta_{1}^{2}$, 2. $a d_{a} \cos \left(d_{a}, A_{2}, a\right)+b \delta_{\beta} \cos \left(\delta_{\beta}, \Delta_{2}, b\right)+c \delta_{\gamma} \cos \left(\delta_{\gamma}, \Delta_{2}, c\right)=\Sigma k^{2}-\Delta_{2}^{2}$, 3. $a \delta_{\alpha} \cos \left(\delta_{\alpha}, A_{3}, a\right)+b d_{b} \cos \left(d_{b}, A_{3}, b\right)+c \delta_{\gamma} \cos \left(\delta_{\gamma}, A_{3}, c\right)=\Sigma k^{2}-\Delta_{3}^{2}$, 4. $a \delta_{\alpha} \cos \left(\delta_{\alpha}, A_{4}, a\right)+b \delta_{\beta} \cos \left(\delta_{\beta}, A_{4}, b\right)+c d_{c} \cos \left(d_{c}, A_{4}, c\right)=\Sigma k^{2}-A_{4}^{2}$.

a. Es ist $d_{a} \cos \left(d_{a}, \mathcal{A}_{1}, a\right)=H B \cos H B A=-G A \cos G A B=-A G_{1}$ $=-\left(\boldsymbol{C}_{\mathbf{1}} \boldsymbol{G}_{\mathbf{1}}+\boldsymbol{A} \boldsymbol{C}_{\mathbf{1}}\right)=-(\boldsymbol{c} \cos \beta+\boldsymbol{b} \cos \gamma)$; wie (83. 1.). Die Ausdrücke (83. 2. u. 3.) folgen aus (83. 1.) nach (\$.15. B.), und (84, 85 u. 86.) folgen aus (83.), vermöge (52.).

b. Die Ausdrücke (87.) folgen aus $(83,84,85,86$, u. 52.).

VII.

Es ist für (\$. 14. g.)

(88.) $\begin{cases}\text { 1. } & \Delta_{1} \cos \left(A_{1}, d_{a}, a\right)=a+c \cos \beta+b \cos \gamma, \\ \text { 2. } & \Delta_{1} \cos \left(A_{1}, d_{a}, b\right)=b+a \cos \gamma+c \cos \alpha,\end{cases}$

3. $\Delta_{1} \cos \left(A_{1}, d_{c}, c\right)=c+b \cos \alpha+a \cos \beta$.

(89.) $\begin{cases}\text { 1. } & \Delta_{2} \cos \left(\Delta_{2}, d_{a}, a\right)=a-c \cos \beta-b \cos \gamma, \\ \text { 2. } & \Delta_{2} \cos \left(\Delta_{2}, \delta_{\beta}, b\right)=b-a \cos \gamma+c \cos \alpha,\end{cases}$

3. $A_{2} \cos \left(A_{2}, \delta_{\gamma}, c\right)=c+b \cos \alpha-a \cos \beta$.

1. $\Delta_{3} \cos \left(A_{3}, \delta_{\alpha}, a\right)=a f-c \cos \beta-b \cos \gamma$,

2. $A_{3} \cos \left(A_{3}, d_{b}, b\right)=b-a \cos \gamma-c \cos \alpha$,

3. $\Delta_{3} \cos \left(A_{3}, \delta_{\gamma}, c\right)=c-b \cos \alpha+a \cos \beta$.

(91.) $\begin{cases}\text { 1. } & \Delta_{4} \cos \left(\Delta_{4}, \delta_{\alpha}, a\right)=a+b \cos \gamma-c \cos \beta, \\ \text { 2. } & \Delta_{4} \cos \left(\Delta_{4}, \delta_{\beta}, b\right)=b-c \cos \alpha+a \cos \gamma, \\ \text { 3. } & \Delta_{4} \cos \left(\Delta_{4}, d_{c}, c\right)=c-a \cos \beta-b \cos \alpha .\end{cases}$

1. $a \cos \left(\Delta_{1}, d_{a}, a\right)+b \cos \left(\Delta_{1}, d_{b}, b\right)+c \cos \left(\Delta_{1}, d_{c}, c\right)=\Delta_{1}$,

2. $a \cos \left(\Delta_{2}, d_{a}, a\right)+b \cos \left(\Delta_{2}, \delta_{\beta}, b\right)+c \cos \left(A_{2}, \delta_{\gamma}, c\right)=\Delta_{2}$,

3. $a \cos \left(A_{3}, \delta_{\alpha}, a\right)+b \cos \left(A_{3}, d_{b}, b\right)+c \cos \left(\Delta_{3}, \delta_{\gamma}, c\right)=\Delta_{3}$,

4. $a \cos \left(\Delta_{4}, \delta_{\alpha}, a\right)+b \cos \left(\Delta_{4}, \delta_{\beta}, b\right)+c \cos \left(\Delta_{4}, d_{c} ; c\right)=A_{4}$. 
a. $\quad E s$ ist $\Delta_{1} \cos \left(\Delta_{1}, d_{a}, a\right)=H A \cos H A B=A H_{1}=A B+B H_{1}$ $=A B+H B \cos H B H_{1}=a+d_{a} \cos H B H_{1}=a+b \cos \gamma+c \cos \beta^{\prime}(64$.$) .$ Dies ist der Ausdruck (88. 1.). Die übrigen in $(88,89,90$ u. 91.) folgen nach (\$. 15. A u. B.).

b. Die Formel (92. 1.) folgt aus (88.) und (58.1.), wenn man (88. 1, 2, 3.) der Reihe nach mit $a, b, c$ multiplicirt und die Summe durch $\Delta_{1}$ dividirt. Die übrigen Formeln (92.) folgen ähnlich aus (89, 90 u. 91.).

c. Die Formeln (92.) sind, wie man sieht, ganz ähnlich denen für Parallelogramme. Denn es ist z. B. in dem Parallelogramm $A B C D$ die Diagonal $\boldsymbol{A D}$ oder $d_{c}=a \cos \left(d_{c}, a\right)+b \cos \left(d_{c}, b\right)$.

\section{VIII.}

Es ist für $(\$ .14 . g$.

$\begin{cases}\text { 1. } & d_{a} \Delta_{1} \cos \left(\Delta_{1}, a, d_{\alpha}\right)=d_{a}^{2}+a c \cos \beta+b a \cos \gamma, \\ \text { 2. } & d_{b} \Delta_{1} \cos \left(\Delta_{1}, b, d_{b}\right)=d_{b}^{2}+b a \cos \gamma+c b \cos \alpha, \\ \text { 3. } & d_{c} \Delta_{1} \cos \left(\Delta_{1}, c, d_{c}\right)=d_{c}^{2}+c b \cos \alpha+a c \cos \beta .\end{cases}$

(94.)

1. $d_{a} A_{2} \cos \left(A_{2}, a, d_{a}\right)=d_{a}^{2}-a c \cos \beta-b a \cos \gamma$,

2. $\delta_{\beta} A_{2} \cos \left(\Delta_{2}, b, \delta_{\beta}\right)=\delta_{\beta}^{2}-b a \cos \gamma+c b \cos \alpha$,

3. $\delta_{\gamma} \Delta_{2} \cos \left(A_{2}, c, \delta_{\gamma}^{\prime}\right)=\delta_{\gamma}^{2}+c b \cos \alpha-a c \cos \beta$.

1. $\delta_{\alpha} A_{3} \cos \left(\Delta_{3}, a, \delta_{\alpha}\right)=\delta_{\alpha}^{2}+a c \cos \beta-b a \cos \gamma$,

(95.) $\quad$ 2. $d_{b} \Delta_{3} \cos \left(\Delta_{3}, b, d_{b}\right)=d_{b}^{2}-b a \cos \gamma-c b \cos \alpha$,

3. $\delta_{\gamma} \Delta_{3} \cos \left(\Delta_{3}, c, \delta_{\gamma}\right)=\delta_{\gamma}^{2}-c b \cos \alpha+a c \cos \beta$.

1. $\delta_{\alpha} A_{4} \cos \left(A_{4}, a, \delta_{\alpha}\right)=\delta_{\alpha}^{2}-a c \cos \beta+b a \cos \gamma$,

(96.)

2. $\delta_{\beta} A_{4} \cos \left(A_{4}, b, \delta_{\beta}\right)=\delta_{\beta}^{2}+b a \cos \gamma-c b \cos \gamma$,

3. $d_{c} A_{4} \cos \left(A_{4}, c, d_{c}\right)=d_{c}^{2}-c b \cos \alpha-a c \cos \gamma$.

(97.)

$\begin{cases}1 . & d_{a} \cos \left(\Delta_{1}, a, d_{a}\right)+d_{b} \cos \left(\Delta_{1}, b, d_{b}\right)+d_{c} \cos \left(\Delta_{1}, c, d_{c}\right)=2 \Delta_{1}, \\ 2 . & d_{a} \cos \left(\Delta_{2}, a, d_{a}\right)+\delta_{\beta} \cos \left(\Delta_{2}, b, d_{\beta}\right)+\delta_{\gamma} \cos \left(\Delta_{2}, c, \delta_{\gamma}\right)=2 \Delta_{2}, \\ 3 . & d_{\alpha} \cos \left(\Delta_{3}, a, \delta_{\alpha}\right)+d_{b} \cos \left(\Delta_{3}, b, d_{b}\right)+\delta_{\gamma} \cos \left(\Delta_{3}, c, \delta_{\gamma}\right)=2 \Delta_{3}, \\ 4 . & \delta_{\alpha} \cos \left(\Delta_{4}, a, \delta_{\alpha}\right)+\delta_{\beta} \cos \left(\Delta_{4}, b, \delta_{\beta}\right)+d_{c} \cos \left(\Delta_{4}, c, d_{c}\right)=2 \Delta_{4} .\end{cases}$

a. Im Dreieck $\boldsymbol{H A B}$ ist $\boldsymbol{H B}=\boldsymbol{A H} \cos \boldsymbol{A H B}+\boldsymbol{A B} \cos H B A$, das heifst, $d_{a}=\Delta_{1} \cos \left(\Delta_{1}, a, d_{a}\right)+a \cos \left(d_{a}, \Delta_{1}, a\right)$. Dies giebt vermöge (83. 1.) $d_{a}=\Delta_{1} \cos \left(\Delta_{1}, a, d_{a}\right)-\frac{a}{d_{a}}(c \cos \beta+b \cos \gamma)$, also $d_{a} \Delta_{1} \cos \left(\Delta_{1}, a, d_{a}\right)$ $=d_{a}^{2}+a c \cos \beta+b a \cos \gamma$; wie (93. 1.). Die übrigen Ausdrücke in (93, 94, 95 und 96.) folgen nach (\$. 15. $A$ und B.). 
15. Vom Parallelogranm- und dem Parallelepipedum der Kräfte.

b. Die Summe von (93. 1,2u. 3.) ist $\Delta_{1}\left[d_{a} \cos \left(A_{1}, a, d_{a}\right)+d_{b} \cos \left(\Delta_{1}, b, d_{b}\right)\right.$ $\left.+d_{c} \cos \left(A_{1}, c, d_{c}\right)\right]=d_{a}^{2}+d_{b}^{2}+d_{c}^{2}+2 a c \cos \beta+2 b \iota \cos \gamma+2 c b \cos \alpha$ (60.) $=\Sigma k^{2}+\Delta_{1}^{2}-\Sigma k^{2}$ (58. 1.) $=2 \Delta_{1}^{2}$; woraus, mit $\Delta_{1}$ dividirt, (97. 1.) folgt. Die übrigen Formeln in (97.) ergeben sich nach (\$.15. A.) und (52.).

IX.

Es ist für $(\$ .14 . g$.$) :$

(98.)

1. $\quad d_{a} d_{b} \cos \left(d_{a}, \delta_{\gamma}, d_{b}\right)=c^{2}+b c \cos \alpha+c a \cos \beta+a b \cos \gamma$,

2. $d_{b} d_{c} \cos \left(d_{b}, \delta_{\alpha}, d_{c}\right)=a^{2}+c a \cos \beta+a b \cos \gamma+b c \cos \alpha$,

3. $\quad d_{c} d_{a} \cos \left(d_{c}, \delta_{\beta}, d_{a}\right)=b^{2}+a b \cos \gamma+b c \cos \alpha+c a \cos \beta$.

1. $d_{a} \delta_{\beta} \cos \left(d_{a}, d_{c}, \delta_{\beta}\right)=c^{2}+b c \cos \alpha-c a \cos \beta-a b \cos \gamma$,

(99.) 2. $\delta_{\beta} \delta_{\gamma} \cos \left(\delta_{\beta}, \delta_{\alpha}, \delta_{\gamma}\right)=a^{2}-c a \cos \beta-a b \cos \gamma+b c \cos \alpha$,

3. $\delta_{\gamma} d_{a} \cos \left(\delta_{\gamma}, d_{b}, d_{a}\right)=b^{2}-a b \cos \gamma+b c \cos \alpha-c a \cos \beta$.

1. $\delta_{\alpha} d_{b} \cos \left(\delta_{\alpha}, d_{c}, d_{b}\right)=c^{2}-b c \cos \alpha+c a \cos \beta-a b \cos \gamma$,

(100.) 2. $\quad d_{b} \delta_{\gamma} \cos \left(d_{b}, d_{a}, \delta_{\gamma}\right)=a^{2}+c a \cos \beta-a b \cos \gamma-b c \cos \alpha$,

3. $\delta_{\gamma} \delta_{\alpha} \cos \left(\delta_{\gamma}, \delta_{\beta}, \delta_{\alpha}\right)=b^{2}-a b \cos \gamma-b c \cos \alpha+c a \cos \beta$.

1. $\delta_{\alpha} \delta_{\beta} \cos \left(\delta_{\alpha}, \delta_{\gamma}, \delta_{\beta}\right)=c^{2}-b c \cos \alpha-c a \cos \beta+a b \cos \gamma$,

(101.) $\quad$ 2. $\delta_{\beta} d_{c} \cos \left(\delta_{\beta}, d_{a}, d_{c}\right)=a^{2}-c a \cos \beta+a b \cos \gamma-b c \cos \alpha$,

3. $d_{c} \delta_{\alpha} \cos \left(d_{c}, d_{b}, \delta_{\alpha}\right)=b^{2}+a b \cos \gamma-b c \cos \alpha-c a \cos \beta$.

1. $d_{a} d_{b} \cos \left(d_{a}, \delta_{\gamma}, d_{b}\right)+d_{b} d_{c} \cos \left(d_{b}, \delta_{\alpha}, d_{c}\right)+d_{c} d_{a} \cos \left(d_{c}, \delta_{\beta}, d_{a}\right)$ $=\frac{3}{2} A_{1}^{2}-\frac{1}{2} \Sigma k^{2}$,

2. $d_{\alpha} d_{\beta} \cos \left(d_{a}, d_{c}, \delta_{\beta}\right)+\delta_{\beta} \delta_{\gamma} \cos \left(\delta_{\beta}, \delta_{\alpha}, \delta_{\gamma}\right)+\delta_{\gamma} d_{a} \cos \left(\delta_{\gamma}, d_{b}, d_{a}\right)$ $=\frac{3}{2} \Delta_{2}^{2}-\frac{1}{2} \Sigma k^{2}$

(102.)

3. $\delta_{\alpha} d_{b} \cos \left(\delta_{a}, d_{c}, d_{b}\right)+d_{b} \delta_{\gamma} \cos \left(d_{b}, d_{a}, \delta_{\gamma}\right)+\delta_{\gamma} \delta_{\alpha} \cos \left(\delta_{\gamma}, \delta_{\beta}, \delta_{\alpha}\right)$

$=\frac{3}{2} \Delta_{3}^{2}-\frac{1}{2} \Sigma k^{2}$

4. $\delta_{\alpha} \delta_{\beta} \cos \left(\delta_{\alpha}, \delta_{\gamma}, \delta_{\beta}\right)+\delta_{\beta} d_{c} \cos \left(\delta_{\beta}, d_{a}, d_{c}\right)+d_{c} \delta_{\alpha} \cos \left(d_{c}, \delta_{b}, \delta_{\alpha}\right)$ $=\frac{3}{2} \Delta_{4}^{2}-\frac{1}{2} \Sigma k^{2}$.

a. In dem Dreieck $\boldsymbol{G} A \boldsymbol{F}=\left(d_{a} \delta_{\gamma} d_{b}\right)$ ist $d_{a}^{2}+d_{b}^{2}-2 d_{a} d_{b} \cos \left(d_{a} \delta_{\gamma} d_{b}\right)$ $=\delta_{\gamma}^{2}$, also nach (53. u. 54.) $b^{2}+c^{2}+2 b c \cos \alpha+c^{2}+a^{2}+2 c a \cos \beta-a^{2}-b^{2}$ $+2 a b \cos \gamma=2 d_{a} d_{b} \cos \left(d_{a} \delta_{\gamma} d_{b}\right)$ oder $c^{2}+b c \cos \alpha+c a \cos \beta+a b \cos \gamma$ $=d_{a} d_{b} \cos \left(d_{a} \delta_{\gamma} d_{b}\right)$; wie (98. 1.). Nach (\$. 15. B.) finden sich (98. 2. u. 3.).

b. In dem Dreieck EBH $=\left(d_{a} d_{c} \delta_{\beta}\right)$ ist $d_{a}^{2}+\delta_{\beta}^{2}-2 d_{\alpha} \delta_{\beta} \cos \left(d_{a} d_{c} \delta_{\beta}\right)$ $=d_{c}^{2}$, also nach (53. u. 54.) $b^{2}+c^{2}+2 b c \cos \alpha+c^{2}+a^{2}-2 c a \cos \beta-a^{2}-b^{2}$ $-2 a b \cos \gamma=2 d_{a} \delta_{\beta} \cos \left(d_{a} d_{c} \delta_{\beta}\right)$ oder $c^{2}+b c \cos \alpha-c a \cos \beta-a b \cos \gamma$ $=d_{a} \delta_{\beta} \cos \left(d_{a} d_{c} \delta_{\beta}\right)$; wie (99. 1.). Nach (\$. 15. B.) finden sich (99. 2. u. 3.). 
c. In dem Dreieck $\boldsymbol{C H E}=\left(\delta_{\alpha} d_{c} d_{b}\right)$ ist $\delta_{\alpha}^{2}+d_{b}^{2}-2 \delta_{\alpha} d_{b} \cos \left(\delta_{\alpha} d_{c} d_{b}\right)$ $=d_{c}^{2}$, also nach (53. u. 54.) $b^{2}+c^{2}-2 b c \cos \alpha+c^{2}+a^{2}+2 c a \cos \beta-a^{2}-b^{2}$ $-2 a b \cos \gamma=2 \delta_{\alpha} d_{b} \cos \left(\delta_{\alpha} d_{c} d_{b}\right)$ oder $c^{2}-b c \cos \alpha+c a \cos \beta-a b \cos \gamma$ $=\delta_{\alpha} d_{b} \cos \left(\delta_{\alpha} d_{c} d_{b}\right)$; wie (100.1.). Nach (\$.15. B.) finden sich (100. 2. u. 3.).

d. In dem Dreieck $\boldsymbol{G D F}=\left(\delta_{\alpha}, \delta_{\gamma}, \delta_{\beta}\right)$ ist $\delta_{\alpha}^{2}+\delta_{\beta}^{2}-2 \delta_{\alpha} \delta_{\beta} \cos \left(\delta_{\alpha}, \delta_{\gamma}, \delta_{\beta}\right)$ $=\delta_{\gamma}^{2}$, also nach (53. u. 54.) $b^{2}+c^{2}-2 b c \cos \alpha+c^{2}+a^{2}-2 c a \cos \beta-a^{2}-b^{2}$ $+2 a b \cos \gamma=2 \delta_{\alpha} \delta_{\beta} \cos \left(\delta_{a}, \delta_{\gamma}, \delta_{\beta}\right)$ oder $c^{2}-b c \cos \alpha-c a \cos \beta+a b \cos \gamma$ $=\delta_{\alpha} \delta_{\beta} \cos \left(\delta_{\alpha}, \delta_{\gamma}, \delta_{\beta}\right)$; wie (101.1.). Nach (\$.15. B.) finden sich (101. 2. u. 3.).

e. Die Ausdrücke (102.) folgen, wie leicht zu sehen, aus $(98,99$, 100, 101 u. 58.).

f. Die Cosinus der andern beiden Winkel in den Dreiecken $\boldsymbol{G A F}$, $\boldsymbol{E B H}, \boldsymbol{C H E}$ und $\boldsymbol{G D F}(\boldsymbol{a}, \boldsymbol{b}, \boldsymbol{c}, \boldsymbol{d})$ finden sich schon in den obigen Ausdrücken; z. B.

$$
\begin{gathered}
\cos \left(\delta_{\gamma}, d_{b}, d_{a}\right) \text { aus }(99.3 .) \text { und } \cos \left(d_{b}, d_{a}, \delta_{\gamma}\right) \text { aus (100. 2.) zu } \\
\cos \left(d_{a}, \delta_{\gamma}, d_{b}\right)(98.1 .),
\end{gathered}
$$

und so die andern.

$$
\text { X. }
$$

Es ist für (\$. 14. i.)

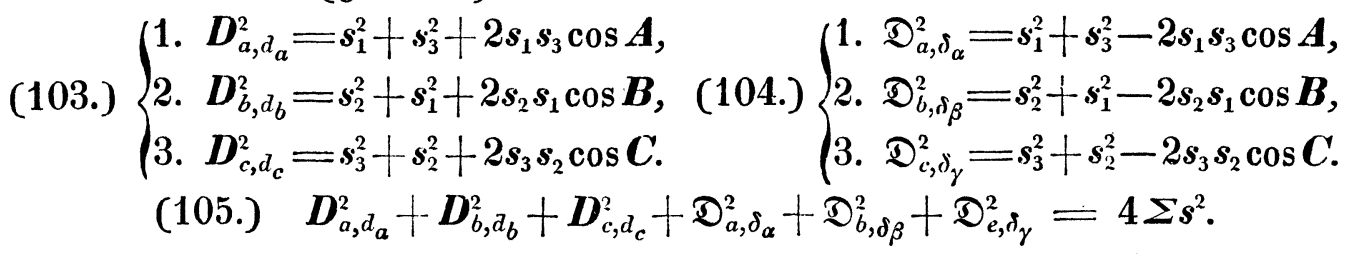

a. Wenn man nemlich auf $\boldsymbol{A B}=\boldsymbol{a}$. eine Ebene $\boldsymbol{G G}_{2} \boldsymbol{G}_{3} \boldsymbol{G}$, die durch $\boldsymbol{G}$ bezeichnet werden mag, senkrecht legt, so steht dieselbe auch auf den mit $\boldsymbol{A B}$ parallelen Linien $\boldsymbol{G H}=\boldsymbol{E H}=\boldsymbol{C D}=\boldsymbol{a}$ senkrecht, und die Linien $\boldsymbol{G} \boldsymbol{G}_{3}=\boldsymbol{c} \sin \beta$ und $\boldsymbol{G}_{3} \boldsymbol{G}_{1}=\boldsymbol{b} \sin \gamma$, in welchen die Ebene $\boldsymbol{G}$ die Ebenen $\boldsymbol{C G H D}=s_{3}\left(\$ .14\right.$. h.) und $\boldsymbol{A B C D}=s_{1}$ schneidet, schliefsen in $\boldsymbol{G}_{3}$ den Winkel $2 \varrho-A$ ein; also ist in $\operatorname{dem}$ Dreieck $\boldsymbol{G G}_{3} \boldsymbol{G}_{1}$ die dritte Seite

(106.) $\quad \boldsymbol{G} G_{1}^{2}=G G_{3}^{2}+G_{3} G_{1}^{2}+2 G G_{3} . G_{3} G_{1} \cos A$

$$
=c^{2} \sin \beta^{2}+b^{2} \sin \gamma^{2}+2 b c \sin \beta \sin \gamma \cos A \text {. }
$$

Hingegen die Linien $G_{2} G_{1}=c \sin \beta$ und $G_{3} G_{1}=b \sin \gamma$, in welchen die Ebene $\boldsymbol{G}$ die Ebenen $\boldsymbol{A B E F}=s_{3}$ (\$. 14. h.) und $\boldsymbol{A B C D}=s_{1}$ schneidet, schliefsen in $\boldsymbol{G}_{1}$ den Winkel $\boldsymbol{A}$ ein; also ist in dem Dreiecke $\boldsymbol{G}_{2} \boldsymbol{G}_{1} \boldsymbol{G}_{3}$ die dritte Seite

$$
\begin{aligned}
G_{2} G_{3}^{2} & =G_{2} G_{1}^{2}+G_{3} G_{1}^{2}-2 G_{2} G_{1} \cdot G_{3} G_{1} \cos A \\
& =c^{2} \sin \beta^{2}+b^{2} \sin \gamma^{2}-2 b c \sin \beta \sin \gamma \cos A
\end{aligned}
$$


15. Vom Parallelogramm- und dem Parallelepipedum der Kräfte.

b. Nun ist $\boldsymbol{G G}_{\mathbf{1}}$ (106.) die Höhe des Parallelogramms $\boldsymbol{A B G H}=\boldsymbol{D}_{a, d_{a}}$ (\$. 14. i.) zu dessen Grundlinie $A B=a$, und $\boldsymbol{G}_{2} \boldsymbol{G}_{3}$ (107.) ist die Höhe

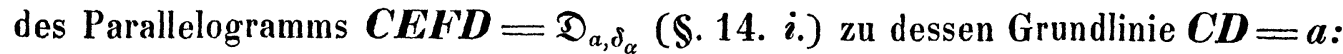
also sind die Quadrate der Inhalte dieser beiden Parallelogramme:

(108.) $\begin{cases}\text { 1. } & D_{a, d_{a}}^{2}=a^{2}\left(c^{2} \sin \beta^{2}+b^{2} \sin \gamma^{2}+2 b c \sin \beta \sin \gamma \cos A\right) \text { und } \\ \text { 2. } & \mathfrak{D}_{a, \delta_{\alpha}}=a^{2}\left(c^{2} \sin \beta^{2}+b^{2} \sin \gamma^{2}-2 b c \sin \beta \sin \gamma \cos A\right),\end{cases}$ welches vermöge (66.)

(109.) $\quad \begin{cases}\text { 1. } & D_{a, d_{a}}^{2}=s_{3}^{2}+s_{1}^{2}+2 s_{3} s_{1} \cos \boldsymbol{A} \text { und } \\ 2 . & \mathfrak{D}_{a, \delta_{a}}^{2}=s_{3}^{2}+s_{1}^{2}-2 s_{3} s_{1} \cos \boldsymbol{A}\end{cases}$ giebt, wie (103. 1. u. 104. 1.).

c. Die andern Ausdrücke (103. u. 104.) folgen nach (\$. 15. B.), und (105.) folgt aus (103. u. 104.) unmittelbar.

XI.

Es ist für $(\$ .14 . k$. und $l$.)

(110.)

$$
\left\{\begin{array}{l}
\text { 1. } 4 \varphi_{1}^{2}=s_{1}^{2}+s_{2}^{2}+s_{3}^{2}-2 s_{1} s_{3} \cos \boldsymbol{A}-2 s_{2} s_{1} \cos \boldsymbol{B}-2 s_{3} s_{2} \cos \boldsymbol{C}^{\prime}, \\
\text { 2. } 4 \varphi_{2}^{2}=s_{1}^{2}+s_{2}^{2}+s_{3}^{2}-2 s_{1} s_{3} \cos \boldsymbol{A}+2 s_{2} s_{1} \cos \boldsymbol{B}+2 s_{3} s_{2} \cos \boldsymbol{C}, \\
\text { 3. } \quad 4 \varphi_{3}^{2}=s_{1}^{2}+s_{2}^{2}+s_{3}^{2}+2 s_{1} s_{3} \cos \boldsymbol{A}-2 s_{2} s_{1} \cos \boldsymbol{B}+2 s_{3} s_{2} \cos \boldsymbol{C}^{\prime}, \\
\text { 4. } \quad 4 \varphi_{4}^{2}=s_{1}^{2}+s_{2}^{2}+s_{3}^{2}+2 s_{1} s_{3} \cos \boldsymbol{A}+2 s_{2} s_{1} \cos \boldsymbol{B}-2 s_{3} s_{2} \cos \boldsymbol{C} .
\end{array}\right.
$$

(111.) $\Sigma \varphi^{2}=\Sigma s^{2}$, das heifst $\varphi_{1}^{2}+\varphi_{2}^{2}+\varphi_{3}^{2}+\varphi_{4}^{2}=s_{1}^{2}+s_{2}^{2}+s_{3}^{2}$.

(112.) $\quad \psi_{1}=\varphi_{1}, \quad \psi_{2}=\varphi_{2}, \quad \psi_{3}=\varphi_{3}, \quad \psi_{4}=\varphi_{4}$.

Die Flächen $\varphi$ sind die vierten Flächen dreieckiger Pyramiden. Z. B. $\varphi_{1}=B C E$ ist die vierte Seitenfläche der dreieckigen Pyramide $A B C E$, deren drei andere Seitenflächen $\boldsymbol{C A B}=\frac{1}{2} s_{1}, C \boldsymbol{A E}=\frac{1}{2} s_{2}$ und $\boldsymbol{E A B}=\frac{1}{2} s_{3}$ sind, und welche die Winkel $\boldsymbol{A}, \boldsymbol{B}, \boldsymbol{C}$ einschliefsen. Die vierte Seitenfläche einer dreieckigen Pyramide wird also nach (110.) durch die drei andern und durch die Winkel, welche dieselben mit einander machen, auf eine ganz ähnliche Weise ausgedrückt, wie die dritte Seite eines Dreiecks durch die beiden andern Seiten und durch den Winkel, welchen sie einschliefsen

a. Die Fläche des Dreiecks $\boldsymbol{B C} \boldsymbol{E}=\varphi_{1}$ ist $\varphi_{1}=\frac{1}{2} \boldsymbol{B C} . \boldsymbol{E} \boldsymbol{E}_{2}=\frac{1}{2} \delta_{\gamma} . \boldsymbol{E} \boldsymbol{E}_{2}$, wenn $\boldsymbol{E} \boldsymbol{E}_{2}$ auf $\boldsymbol{C B}$ senkrecht ist. Nun ist $\gamma^{\prime}\left(\boldsymbol{C} \boldsymbol{E}^{2}-\boldsymbol{E} \boldsymbol{E}_{2}^{2}\right)+\sqrt{ }\left(\boldsymbol{E B} \boldsymbol{B}^{2}-\boldsymbol{E} \boldsymbol{E}_{2}^{2}\right)$ $=\boldsymbol{C B}$, das heifst $\sqrt{ }\left(\delta_{\alpha}^{2}-\boldsymbol{E} \boldsymbol{E}_{2}^{2}\right)+\gamma\left(\delta_{\beta}^{2}-\boldsymbol{E} \boldsymbol{E}_{2}^{2}\right)=\delta_{\gamma}$. Daraus folgt $\delta_{\alpha}^{2}-\boldsymbol{E} \boldsymbol{E}_{2}$ $=\delta_{\gamma}^{2}+\delta_{\beta}^{2}-\boldsymbol{E} \boldsymbol{E}_{2}^{2}-2 \delta_{\gamma} \gamma\left(\delta_{\beta}^{2}-\boldsymbol{E} \boldsymbol{E}_{2}^{2}\right)$ oder $\delta_{\beta}^{2}+\delta_{\gamma}^{2}-\delta_{\alpha}^{2}=2 \delta_{\gamma} \gamma\left(\delta_{\beta}^{2}-\boldsymbol{E} \boldsymbol{E}_{2}^{2}\right)$ oder $4 \delta_{\gamma}^{2} \boldsymbol{E} \boldsymbol{E}_{2}^{2}=4 \delta_{\gamma}^{2} \delta_{\beta}^{2}-\left(\delta_{\beta}^{2}+\delta_{\gamma}^{2}-\delta_{\alpha}^{2}\right)^{2}$ oder, da $\varphi_{1}=\frac{1}{2} \delta_{\gamma} \boldsymbol{E} \boldsymbol{E}_{2}$ war,

(113.) $16 \varphi_{1}^{2}=4 \delta_{\beta}^{2} \delta_{\gamma}^{2}-\left(\delta_{\beta}^{2}+\delta_{\gamma}^{2}-\delta_{\alpha}^{2}\right)^{2}$. 
15. Vom Parallelogramm- und dem Parallelepipedum der Kräfle.

b. Hierin die Ausdrücke (54.) gesetzl, giebt $16 \varphi_{1}^{2}=4\left(c^{2}+a^{2}-2 c a \cos \beta\right)\left(a^{2}+b^{2}-2 a b \cos \gamma\right)$

$-\left(2 a^{2}-2 c a \cos \beta-2 a b \cos \gamma+2 b c \cos \alpha\right)^{2}$, oder $4 \varphi_{1}^{2}=c^{2} a^{2}+c^{2} b^{2}-2 c^{2} a b \cos \gamma+a^{4}+a^{2} b^{2}-2 a^{3} b \cos \gamma-2 a^{3} c \cos \beta$

$-2 b^{2} c a \cos \beta+4 a^{2} b c \cos \beta \cos \gamma-\left(a^{4}+c^{2} a^{2} \cos \beta^{2}+a^{2} b^{2} \cos \gamma^{2}+b^{2} c^{2} \cos \alpha^{2}\right.$ $-2 a^{3} c \cos \beta-2 a^{3} b \cos \gamma-2 a^{2} b \cos \alpha+2 a^{2} b c \cos \beta \cos \gamma+2 c^{2} a b \cos \alpha \cos \beta$ $\left.+2 b^{2} a c \cos \alpha \cos \gamma\right)$ oder

$$
\begin{array}{r}
4 \varphi_{1}^{2}=a^{2} b^{2} \sin \gamma^{2}+b^{2} c^{2} \sin \alpha^{2}+c^{2} a^{2} \sin \beta^{2}-2 c^{2} a b(\cos \gamma-\cos \alpha \cos \beta) \\
-2 a^{2} b c(\cos \alpha-\cos \beta \cos \gamma)-2 b^{2} c \boldsymbol{c}(\cos \beta-\cos \gamma \cos \alpha)
\end{array}
$$

oder zufolge (67.)

$$
4 \varphi_{1}^{2}=a^{2} b^{2} \sin \gamma^{2}+b^{2} c^{2} \sin \alpha^{2}+c^{2} a^{2} \sin \beta^{2}
$$

$-2 c^{2} a b \sin \alpha \sin \beta \cos C-2 a^{2} b c \sin \beta \sin \gamma \cos A-2 b^{2} c a \sin \gamma \sin \alpha \cos B$,

und dies giebt zufolge (66.) den Ausdruck (110.1.). Die andern Ausdrücke (110.) ergeben sich nach (\$.15.), und (111.) folgt unmiltelbar aus (110.).

c. Die Seiten z. B. des Dreiecks $\varphi_{1}=\boldsymbol{B C E}$ sind denen des Dreiecks $\psi_{1}=\boldsymbol{G D F}$ gleich, nämlich $\boldsymbol{G} \boldsymbol{F}=\boldsymbol{C B}=\delta_{\gamma}, \boldsymbol{D F}=\boldsymbol{C E}=\delta_{\alpha}$ und $\boldsymbol{G D}$ $=\boldsymbol{E B}=\delta_{\beta}$, also sind die Dreiecke einander gleich und es ist folglich $\varphi_{1}=\psi_{1}$ u. s. w.; wie (112.).

\section{XII.}

Es ist für $\left(\$_{i} 14 . g.\right)$ :

(115.)

$$
\begin{aligned}
& \text { 1. } c \sin \beta \sin A=c \sin \left(c, s_{1}\right)=d_{a} \sin \left(d_{a}, s_{1}\right)=d_{b} \sin \left(d_{b}, s_{1}\right) \\
& =\delta_{\alpha} \sin \left(\delta_{\alpha}, s_{1}\right)=\delta_{\beta} \sin \left(\delta_{\beta}, s_{1}\right) \\
& =\Delta_{1} \sin \left(\Delta_{1}, s_{1}\right)=\Delta_{2} \sin \left(A_{2}, s_{1}\right)=\Delta_{3} \sin \left(\Delta_{3}, s_{1}\right)=\Delta_{4} \sin \left(\Delta_{4}, s_{1}\right), \\
& \text { 2. } \quad a \sin \gamma \sin B=a \sin \left(a, s_{2}\right)=d_{b} \sin \left(d_{b}, s_{2}\right)=d_{c} \sin \left(d_{c}, s_{2}\right) \\
& =\delta_{\beta} \sin \left(\delta_{\beta}, s_{2}\right)=\delta_{\gamma} \sin \left(\delta_{\gamma}, s_{2}\right) \\
& =\Delta_{1} \sin \left(\Delta_{1}, s_{2}\right)=A_{2} \sin \left(A_{2}, s_{2}\right)=\Delta_{3} \sin \left(\Delta_{3}, s_{2}\right)=\Delta_{4} \sin \left(\Delta_{4}, s_{2}\right) \text {, } \\
& \text { 3. } \quad b \sin \alpha \sin C=b \sin \left(b, s_{3}\right)=d_{c} \sin \left(d_{c}, s_{3}\right)=d_{a} \sin \left(d_{a}, s_{3}\right) \\
& =\delta_{\gamma} \sin \left(\delta_{\gamma}, s_{3}\right)=\delta_{\alpha} \sin \left(\delta_{\alpha}, s_{3}\right) \\
& =\Delta_{1} \sin \left(\Delta_{1}, s_{3}\right)=\Delta_{2} \sin \left(\Delta_{2}, s_{3}\right)=\Delta_{3} \sin \left(\Delta_{3}, s_{3}\right)=\Delta_{4} \sin \left(\Delta_{4}, s_{3}\right) \text {. }
\end{aligned}
$$

a. Denn von allen den Linien $c=A \boldsymbol{E}, d_{a}=A \boldsymbol{G}, d_{b}=\boldsymbol{A F}$, $\delta_{\alpha}^{\prime}=\boldsymbol{C E}, \delta_{\beta}=\boldsymbol{B E}, \Delta_{1}=\boldsymbol{A H}, \Delta_{2}=\boldsymbol{B G}, \Delta_{3}=\boldsymbol{C F}$ und $\Delta_{4}=\boldsymbol{E D}$ liegt der eine Endpunct in der einen der beiden parallelen Seitenflächen $\boldsymbol{A B C D}$ $=\boldsymbol{E F G H}=s_{1}$, der andere in der andern. Daher ist das Product jeder 
dieser Linien in den Sinus des Winkels, welchen sie mit einer der beiden Seitenflächen $s_{1}$ einschliefst, gleich der senkrechten Entfernung der beiden Seitenflächen von einander; und diese Entfernung ist $\boldsymbol{E} \boldsymbol{A} \cdot \sin \boldsymbol{E} \boldsymbol{A B} \cdot \sin \boldsymbol{A}$ $=c \sin \beta \sin A$. Daraus folgt also (115. 1.). Die andern Ausdrücke (115. 2. u. 3.) folgen daraus nach (\$.15.).

XIII.

Es ist für $(\$ .14 .9$.$) :$

$$
\text { (116.) } \quad \begin{cases}\text { 1. } & \boldsymbol{D}_{a, d_{a}} \sin \left(s_{1}, \boldsymbol{D}_{a, d_{a}}\right)=s_{3} \sin \boldsymbol{A}=\mathfrak{D}_{a, \delta_{\alpha}} \sin \left(s_{1}, \mathfrak{D}_{a, \delta_{\alpha}}\right), \\ \text { 2. } & \boldsymbol{D}_{b, d_{b}} \sin \left(s_{2}, \boldsymbol{D}_{b, d_{b}}\right)=s_{1} \sin \boldsymbol{B}=\mathfrak{D}_{b, \delta_{\beta}} \sin \left(s_{2}, \mathfrak{D}_{b, \delta_{\beta}}\right), \\ \text { 3. } & \boldsymbol{D}_{c, d_{c}} \sin \left(s_{3}, \boldsymbol{D}_{c, d_{c}}\right)=s_{2} \sin \boldsymbol{C}=\mathfrak{D}_{c, \delta_{\gamma}} \sin \left(s_{3}, \mathfrak{D}_{c, \delta_{\gamma}}\right) .\end{cases}
$$

a. Denn legt man durch $\boldsymbol{A B}=a$ eine auf $\boldsymbol{A B C D}=s_{1}$ senkrechte Ebene $\boldsymbol{E}$ und projicirt auf dieselbe die Ebene $\boldsymbol{A B E F}=s_{3}$, so ist die Länge der parallelogrammatischen Projection $=a$, und ihre Höhe ist gleich dem senkrechten Abstande der beiden Ebenen $\boldsymbol{A B C D}=s_{1}$ und $\boldsymbol{E F G H}=s_{1}$ von einander, der, wie in (XII.), $=c \sin \beta \sin \boldsymbol{A}$ ist: also ist der Flächen-Inhalt der Projection $=a c \sin \beta \sin \boldsymbol{A}=s_{3} \sin \boldsymbol{A}$.

b. Dieselbe Länge und dieselbe Höhe haben aber auch die parallelo-

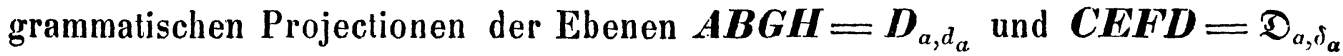
auf die Ebene $\boldsymbol{E}$, die durch $\boldsymbol{D}_{a, d_{a}} \sin \left(\boldsymbol{s}_{1}, \boldsymbol{D}_{a, d_{a}}\right)$ und $\mathfrak{D}_{a, \delta_{\alpha}} \sin \left(\boldsymbol{s}_{1}, \mathfrak{D}_{a, \delta_{\alpha}}\right)$ ausgedrückt werden. Also sind ihre Flächen-Inhalte $s_{3} \sin A$ dem der Projection von $s_{3}$ gleich; was (116. 1.) giebt.

Die andern. Ausdrücke (116. 2 u. 3.) ergeben sich nach (\$.15.).

XIV.

Der körperliche Inhalt des Parallelepipedums $\boldsymbol{A H}(\$ .14 . m)$ wird durch 1. $\boldsymbol{P}=a b c \sin \beta \sin \gamma \sin \boldsymbol{A}=a b c \sin \gamma \sin \alpha \sin \boldsymbol{B}$ $=a b c \sin \alpha \sin \beta \sin C$ und

(117.)

2. $\boldsymbol{P}=a b c \lambda=$ $a b c \sqrt{ }\left(1-\cos \alpha^{2}-\cos \beta^{2}-\cos \gamma^{2}+2 \cos \alpha \cos \beta \cos \gamma\right)$ (51.) oder 3. $\boldsymbol{P}=2 a b c_{1} /\left[\sin \frac{1}{2}(\alpha+\beta+\gamma) \sin \frac{1}{2}(\alpha+\beta-\gamma) \sin \frac{1}{2}(\alpha-\beta+\gamma)\right.$ $\left.\times \sin \frac{1}{2}(-\alpha+\beta+\gamma)\right](51$.$) und$

4. $\boldsymbol{p}=-4 a b c \cdot \frac{\cos \frac{1}{2}(A+B+C) \cos \frac{1}{2}(A+B-C) \cos \frac{1}{2}(B+C-A) \cos \frac{1}{2}(C+A-B)}{\sin A \sin B \sin C}$ ausgedrückt. 
258 15. Vom Parallelogramm- und dem Parallelepipedum der Kräfte.

a. Es ist $\boldsymbol{P}$ gleich der Fläche $\boldsymbol{A B C D}=s_{1}=a b \sin \gamma(66$.$) , mul-$ tiplicirt mit der senkrechten Höhe $e=c \sin \beta \sin A$ (XII.) des Puncts $\boldsymbol{E}$ über $\boldsymbol{A B C D}$; also ist $\boldsymbol{P}=a b c \sin \beta \sin \gamma \sin \boldsymbol{A}$, und nach (\$.15.) $=a b c \sin \gamma \sin \alpha \sin \boldsymbol{B}$ $=a b c \sin \alpha \sin \beta \sin C$; wie (117. 1.).

b. Nach (72.) ist $\lambda=\sin \beta \sin \gamma \sin A$; woraus (117. 2.) folgt.

c. (117. 3.) hat den andern Ausdruck von $\lambda$ (51.).

d. Es ist

(118.)

$\left\{\begin{array}{l}\sin \beta=\frac{2 \sqrt{ }\left(-\cos \frac{1}{2}(A+B+C) \cos \frac{1}{2}(A+B-C) \cos \frac{1}{2}(B+C-A) \cos \frac{1}{2}(C+A-B)\right)}{\sin A \sin C} \\ \sin \gamma=\frac{2 \sqrt{ }\left(-\cos \frac{1}{2}(A+B+C) \cos \frac{1}{2}(A+B-C) \cos \frac{1}{2}(B+C-A) \cos \frac{1}{2}(C+A-B)\right)}{\sin B \sin A} .\end{array}\right.$

Man findet diese Ausdrücke z. B. in meinem Lehrbuche der Geometrie (2. Theil S. 904. 58. u. 59.). Es folgt daraus, wenn man nach (117. 1.) $\sin \beta \sin \gamma$ mit $\sin \boldsymbol{A}$ und $a b c$ multiplicirt, der Ausdruck (117. 4.) von $\boldsymbol{P}$.

\section{XV.}

Die vier Raum-Diagonalen schneiden sich in einem und demselben Punct $\boldsymbol{M}$, der sie alle halbirt und von je zwei parallelen Seitenflächen des Parallelepipedums gleich weit entfernt ist.

a. Die beiden Raum-Diagonalen $\boldsymbol{A H}=\Delta_{1}$ und $\boldsymbol{B G}=\Delta_{2}$ sind die beiden Diagonalen des Parallelogramms $\boldsymbol{A B G H}$ und halbiren also einander in $\boldsymbol{M}$. Ferner sind $\boldsymbol{A H}=\Delta_{1}$ und $\boldsymbol{C F}=\Delta_{3}$ die beiden Diagonalen des Parallelogramms $\boldsymbol{A C H}$, und folglich halbirt der Punct $\boldsymbol{M}$, in der Mitte von $\boldsymbol{A H}=\mathcal{A}_{1}$, auch $\boldsymbol{C H}=\mathcal{A}_{3}$. Endlich sind $\boldsymbol{B G}=\mathcal{A}_{2}$ und $\boldsymbol{E D}=\mathcal{A}_{4}$ die beiden Diagonalen des Parallelogramms EGDB : also halbirt der Punct $\boldsymbol{M}$, in der Mitte von $\boldsymbol{B} \boldsymbol{G}=\Delta_{2}$, auch $\boldsymbol{E} \boldsymbol{D}=\Delta_{4}$, und folglich halbirt ein- und derselbe Punct $\boldsymbol{M}$ alle vier Raum-Diagonalen.

b. Dafs $\boldsymbol{M}$ von je zwei parallelen Seitenflächen des Parallelepipedums gleich weit entfernt ist, folgt hieraus unmittelbar.

\section{XVI.}

Die Ebenen $\varphi_{1}$ und $\psi_{1}\left(\$ .14 . k\right.$ u. l.) theilen die Raum-Diagonal $\Delta_{1}$ durch ihre Durchschnitte mit ihr in drei gleiche Theile. Eben so die Ebenen $\varphi_{2}$ und $\psi_{2}$ die Diagonal $A_{2}$, die Ebenen $\varphi_{3}$ und $\psi_{3}$ die Diagonal $A_{3}$ und die Ebenen $\varphi_{4}$ und $\psi_{4}$ die Diagonal $A_{4}$. 
a. Es ist $\boldsymbol{G F}$ mit $\boldsymbol{B C}, \boldsymbol{G D}$ mit $\boldsymbol{B E}$ und $\boldsymbol{D F}$ mit $\boldsymbol{C E}$ im Raume parallel: also sind die Ebenen $\boldsymbol{G D F}=\psi_{1}$ und $\boldsymbol{B C E}=\varphi_{1}$ parallel.

b. Nun werden die Diagonalen $\boldsymbol{A G}=\boldsymbol{H B}=\boldsymbol{d}_{a}$ der Seitenflächen $\boldsymbol{A C G E}=\boldsymbol{B D H F}$ von den andern Diagonalen $\boldsymbol{C E}=\boldsymbol{D F}=\delta_{\alpha}$ eben dieser Seitenflächen in $N_{1}$ und $L_{1}$ halbirt. Also ist

$$
\text { (119.) } A N_{1}=N_{1} G=B L_{1}=L_{1} H \text {, }
$$

und auch die Linien $B N_{1}$ und $G L_{1}$ sind parallel.

c. Ferner liegen die Linien $B N_{1}$ und $G L_{1}$ in den parallelen Ebenen $B C E$ und $\boldsymbol{G D F}$, und zugleich in der Ebene $\boldsymbol{A B G H}$, denn $\boldsymbol{N}_{1}$ und $L_{1}$ liegen in derselben. Desgleichen liegt $\boldsymbol{A H}$ in der Ebene $\boldsymbol{A B G H}$. Also schneidet $\boldsymbol{A H}$ die Linien $B N_{1}$ und $G L_{1}$ in $N$ und $L$. Nun sind die Dreiecke $A N_{1} N$ und $\boldsymbol{A G L}$, so wie $H L_{1} L$ und $H N B$, ähnlich: folglich ist, wegen (119.), auch

$$
\text { (120.) } \boldsymbol{A N}=\mathbf{N L}=\boldsymbol{H L} \text {, }
$$

und folglich theilen die Ebenen $\varphi_{1}=\boldsymbol{C E B}$ und $\psi_{1}=\boldsymbol{G} \boldsymbol{D F}$ die Raumdiagonal $\Delta_{1}=A H$ durch ihre Durchschnitte mit ihr in drei gleiche Theile. (An diesen Satz hat mich Herr Prof. J. Steiner erinnert.)

d. Da die Winkel $\alpha, \beta, \gamma$ jede beliebige Gröfse haben können, insofern nicht $\alpha+\beta+\gamma>4 \varrho$ ist, so gilt Dasselbe, was für die Ecke $\boldsymbol{A}$ Statt findet, auch für die Ecken $\boldsymbol{B}, \boldsymbol{C}$ und $\boldsymbol{D}$, und folglich eben Das, was für $\varphi_{1}, \psi_{1}$ und $\Delta_{1}$ Statt findet, auch für $\varphi_{2}, \psi_{2}, \Delta_{2} ; \varphi_{3}, \psi_{3}, \Delta_{3}$ und $\varphi_{4}, \psi_{4}$ und $A_{4}$.

\section{XVII.}

Es ist für (\$. 14. n. u. o.)

$$
\begin{aligned}
& \text { (121.) } \quad p_{1}=p_{2}=p_{3}=p_{4}=\frac{1}{6} \boldsymbol{P}, \\
& \text { (122.) } \quad q_{1}=q_{2}=q_{3}=q_{4}=\frac{1}{3} \boldsymbol{P}, \\
& \text { (123.) } \quad \Sigma p=\frac{2}{3} \boldsymbol{P}, \quad \Sigma q=\frac{4}{3} \boldsymbol{P} .
\end{aligned}
$$

a. Die Pyramide $p_{1}=A B C E$ hat die halbe Seiten-Fläche $A B C=\frac{1}{2} s_{1}$ zur Grundfläche und zur Höhe dieselbe senkrechte Höhe des Puncts $\boldsymbol{E}$ über der Grundfläche, welche, mit der ganzen Fläche $s_{1}$ multiplicirt, den körperlichen Inhalt $\boldsymbol{P}$ des Parallelepipedums giebt. Daher ist $\boldsymbol{p}_{1}=\frac{1}{6} \boldsymbol{P}$; wie (120.). Eben so verhält es sich mit $p_{2}, p_{3}, p_{4}$.

b. Die Grundfläche $\boldsymbol{G D F}=\varphi_{1}$ der Pyramide $\boldsymbol{q}_{1}=\boldsymbol{A G D F}$ ist der Grundfläche $\boldsymbol{B C E}=\psi_{1}$ gleich (112.). Die beiden Flächen $\varphi_{1}$ und $\psi_{1}$ sind parallel (XVI. a.). Ein Perpendikel aus $\boldsymbol{A}$ auf $\varphi_{1}$ gezogen, ist also auch 
260 15. Kom Parallelogramm- und dem Parallelepipedum der Krïfte.

auf $\psi_{1}$ senkrecht und eine und dieselbe gerade Linie. Also bildet er mit $\boldsymbol{A N L}$ einen gleichen Winkel, und folglich verhält sich der Perpendikel aus $\boldsymbol{A}$ auf $\varphi_{1}$ zu dem aus $\boldsymbol{A}$ auf $\psi_{1}$, wie $\boldsymbol{A N}$ zu $\boldsymbol{A L}$, das heifst, wie 1 zu 2 (120.). Demnach ist die Pyramide $q_{1}$ doppelt so grofs als die $p_{1}$, und folglich $=\frac{1}{2} P$ (wie 122.). Eben so verhält es sich mit $q_{2}, q_{3}$ und $q_{4}$.

c. Die Ausdrücke (123.) folgen unmittelbar aus (121 u. 122.).

Es giebt noch mancherlei andere zu bemerkende geometrische Eigenschaften eines Parallelepipedums. Doch mag es an dem Obigen genügen.

Berlin, im April 1852. 